\title{
Clarifications for the Published Article: "A Solution to the Famous Twin's Problem" in the APM of SCIRP at 24 September of 2019
}

\author{
Prodromos Char. Papadopoulos \\ 1st Gymnasium in Giannitsa, Giannitsa, Greece \\ Email: starsee@outlook.com.gr
}

How to cite this paper: Papadopoulos, P.C. (2020) Clarifications for the Published Article: "A Solution to the Famous Twin's Problem" in the APM of SCIRP at 24 September of 2019. Advances in Pure Mathematics, 10, 547-587.

https://doi.org/10.4236/apm.2020.109035

Received: July 9, 2020

Accepted: September 26, 2020

Published: September 29, 2020

Copyright $\odot 2020$ by author(s) and Scientific Research Publishing Inc. This work is licensed under the Creative Commons Attribution International License (CC BY 4.0).

http://creativecommons.org/licenses/by/4.0/

(c) (i) Open Access

\begin{abstract}
This article B is almost autonomous because it can be read independently from the first published article A [1] using only a few parts of the article A. Below are given instructions so to need the reader study only on few places of the article A. Also, in the part A of Introduction, here, you will find simple and useful definitions and the strategy we are going to follow as well useful new theorems (also and in Section 5, which have been produced in this solution). So the published solution of twin's problem can now be easily understood. The inequalities (4.17), (4.18) of Article A are proved here in Section 4 by a new clear method, without the possible ambiguity of the text between the relations (4.14), (4.16) of the Article A. Also we complete the proof for the twin's distribution which we use. At the end here are presented the Conclusions, the Nomenclatures and the numerical control of the proof, which is probably useful as well in coding methods. For a general and convincing picture is sufficient, a study from the beginning of this article B until the end of the part A of the Introduction here as well a general glance on the Section 5 and on the Conclusions below.
\end{abstract}

\section{Keywords}

Twin Problem, Twin's Problem, Unsolved Mathematical Problems, Prime Number Problems, Millennium Problems, Riemann Hypothesis, Riemann's Hypothesis, Number Theory, Information Theory, Probabilities, Statistics

\section{About Study}

This article B is an important development of $\mathrm{A}$ and it does take much time of work together with the A, [1] [2]. The two articles in collaboration complete the solution, so that the solution is convincing. The $\mathrm{B}$ also presents new 
ideas, such as the theory of silver bands. You can study this article B using as a quick update the following sections of the first article A: From the beginning until relation (2.1), from the relation (2.7) until the relation (2.9), from the beginning of Section 3 until the relation (4.14) of the Section 4, and as well from the relation (4.16) until the relation (4.19), from the relation (4.20) until the relation (4.22) of the Section 4. These last two parts also exist in this article B at Section 5 with an important improvement.

For explanations my email is: starsee@outlook.com.gr.

\section{Introduction}

\section{A) Definitions and examples}

We accept the phenomenon or "event" in the probability and information theory as fundamental, defining the event in general as an experiential experience. The events can form sets by their common true propositions (properties) of definition of these sets and distinct each other in every such set by their not common properties. Relying constantly on the power of definition, the events, with their relations as other new events, can define entities of sets. For example the multiverse as an infinite set that probably is reflected onto the infinite information of relations of natural numbers, which includes the events of the known universe as well the infinite others, more strange, potentially events (e.g. infinite events probably in quantum entanglement or not) in a mental information unconscious tank.

Rieman Hypothesis $(\mathrm{RH})$ could reveal that the twins obey in distribution which comes from the spesial distribution of prime numbers, which is statistically equivalent to random choices from an ideal "roulette" which has been regulated to obey to the statistics which is changed from one silver interval to the next exclussively according to the known theorem of logarithmic dilution of prime numbers. The statistics of a common roulette depends on the probability $1 / 36$ for every its number due to equal "statistical influences on 36 numbers" thanks to roulette's symmetries. Similarely, in problems of predictions concern prime numbers, we can make statistical calculations based on the general (catholic) properties coming from equal distributions of the multiples of every prime number. If $\mathrm{RH}$ is valid then the distribution of prime numbers makes the statistical calculation of twins here as well valid. Because we don't know yet if the RH is true, in Section 3 and in the beggining of the Section 5, we will prove the main charachteristics of this statistics (which are necessery for the final proof) on the basis of "the deletions on candidates prime numbers from the multiples of the prime numbers". Based on the power of the mathematical definitions and their accompanies examples we will make the clarification of the final proof which must consist from all necessary propositions for agreement in communication.

We'll start with the definition of the probability of the appearance of a twin in a silver interval: 


$$
\delta_{M}=\left[q_{M}^{2}, q_{M+1}^{2}\right)
$$

We know the information definition of Shanon [3]: $I=\log _{2}(1 / p)$.

This definition tells us that the probability of an event is the other face of the information that is essentially the same natural magnitude. For example, throwing an ideal coin with $p_{1}=p_{2}=1 / 2$ for the two possible events, the information we gain after the result and the entropy before the result will be:

$$
I=\log _{2}[1 /(1 / 2)]=1 \text { bit }=(1 / 8) \text { byte and } S=\sum_{i=1}^{i=2} p_{i} \log _{2}\left[1 / p_{i}\right]=1 \text { bit }
$$

A1) The probability of occurrence of an event $e_{i}$ defined on the elements $a_{n}$ of a set $A$ by definition must be calculated based on this definition.

A2) Let be define the event of not-divisibility $e_{v}(s)=$ "the natural number $v \in \delta_{M}$ is not divisible by the $q_{s} \in \delta_{M}=\left\{q_{1}, q_{2}, \cdots, q_{M}\right\}$ ". In Section 3 of article A we saw that we can define a prime number $q_{a}$ in the $\delta_{M}$ as event $q_{a}=" v=$ prime $^{\prime}=e_{v}(1) \cap e_{v}(2) \cap \cdots \cap e_{v}(M)$. In section 3 and 5 of article $\mathrm{B}$ we will show that two such events $v=q_{a}, \mu=q_{b}, \mu, v \in \delta_{M}$ could be considered in calculations as independent events of each other, symbolically $q_{a}<>q_{b}$, in random $\delta_{M}$ because we will prove in Sections 3, 5 of this article B here that it is valid $e_{v}(s)<>e_{\mu}(s)$ for the pair $e_{v}(s), e_{\mu}(s)$ which defines in random $\delta_{M}$ the events $q_{a}, q_{b}$ by the previous definition of " $v=$ prime". Therefore in our special calculations we can consider that each $v \in \delta_{M}$ will have the same probability $P_{M 0}=P(v)=$ const of being a prime number in the same random $\delta_{M}$. This constant probability $P_{M 0}$ in $\delta_{M}$ can be given by a general relation (80) in below Section 4 .

A3) Therefore, by defining the probability $P_{M 0, t w i n}$ of a twin by the next relation (3) [based on the events of twin's definition], because of the (3) and previous constancy of $P_{M 0}$ in random RSI we have that the $P_{M 0, t w i n}$ will be also constant for each $v \in \delta_{M}$. Therefore these imply the constancy of twin's probability $P_{M 0 \text {,twin }}$ in the random RSI $\delta_{M}$, which we will use for the twins multitude calculation in the Section 5 below. Thus, by counting the elements in a random RSI $\delta_{M}$, the number of twins will be the sum: $\sum_{i=1}^{i=d_{M}} P_{M 0, t w i n}$. We point out that for this sum when we add the $P(i-1, i+1)=P_{M 0, t w i n}$ in a RSI (or $P_{M \text {,twin }}$ in VSI) we add in every position $i$ the presentence of a twin for the place $(i-1, i+1)$. Proposition: (2).

The exactly calculations must be based on this definition. Specifically in counting $N-1$ steps between the natural numbers of a silver interval, if $P(v)$ is the probability (or otherwise frequency) of occurrence of a prime number at a random position $v$ then the total probability that this probable prime number is accompanied by another probable prime number at the position $v+2$, under the conditions of the relation (2.1) in the article A, will be [4]:

$$
P_{M 0, t w i n}=P(v) P(v+2 \| v)=\xi_{M 0}[P(v)]^{2}<[P(v)]^{2}=p
$$

Especially for the above is: $P(v+2 \| v)=\xi_{M} P(v)$. As we will analyze little 
below the factor $\xi_{M}$ satisfies the condition $1>\xi_{M 0}>1 / 2$. If one claims that according to the relation (2.1) of the article A putting as $P(v)$ for the second part of (1.2) then we must put $P(v+2 \| v)$ as $P(v) /(1-0.5)$ because the probable first prime number $v$ gives the information that the other natural number $v+2$ not be divided by 2 , then as we can see from the Section 5 below the our proof continues to be valid, but this claim is not the correct. Proposition: (4).

The reason is that the probability which is gave from (2.1) or every other similar equation [as they are below the (24), (25), (80)-(82), etc.] is constant inside random silver interval as we analyzed below (and as the arithmetical results confirm). On the other hand, the existence of a prime at a position $K$ could similarly give information to any position $K+2 m$ and not only to $K+2$ position, but obviously this does not exist in the proof of (2.1) of article A, and therefore this does not apply.

To see how the relation (3) works cumulatively, suppose we are going to randomly place 3 eggs in the positions of a straight egg cup of 5 consecutive positions. It is logical that there will always be 2 vacancies. Let us also define as a twin the placement of two eggs in two consecutive (successive) positions. The question is: "How many twins are on average expected"? There are two ways to calculate the average number of twins:

1) There are $N_{(m)}=5_{(3)}=(5 !) /(3 ! 2 !)=10$ different ways with a total of 12 twins. Specifically there are 3 ways where the eggs are placed in succession forming 2 twins in each way and so there are $3 \times 2=6$ twins. There is also a way that no twins (0) are formed. Also exist and others 6 ways where 1 twin is formed in each way of these 6 ways and so there are $6 \times 1=6$ twins. Therefore the expected number of twins on average is:

$$
n_{t}=(6+0+6) / 10=12 / 10=6 / 5 .
$$

2) The other calculation method can be based on the relation (3). Let be $s \in\{1,2, \cdots, 10\}$ a random case of putting randomly the $n=3$ eggs in the $N=5$ positions. What is the expected number of twins? The 5 consecutive positions of the egg cup mark $N-1=5-1=4$ boundaries between them. For such random boundary $b \in\{1,2,3,4\}$ (with two positions at left and at right of $b$ let be these "first and second") any egg has $P(v)=n / N=3 / 5$ probability to existing in its first position of $b$. Therefore we conclude that the probability to found in its second neighboring position of $b$ any one of the other $n-1=2$ eggs, so defining a twin of two eggs on their common boundary $b$, will be $P(v+1 \| v)=(n-1) /(N-1)=2 / 4$. The reason is that after the first choice that concerns this boundary $b$ are remaining $n-1=2$ eggs inside the other $N-1=4$ positions. Based on the relation (3) we conclude that the probability to exist one twin of successive eggs for any random boundary $b$ will be:

$$
P_{M 0, t w i n}=P(v) P(v+1 \| v)=(3 / 5)(2 / 4)=6 / 20
$$

Therefore counting the $N-1=4$ successive boundaries we will have the sum 
of four $P_{M 0, t w i n}$, that is the same result of the previous method (1):

$$
n_{t}=\sum_{i=1}^{i=N-1} P_{M 0, t w i n}=\sum_{i=1}^{i=N-1} \frac{n}{N} \frac{n-1}{N-1}=(N-1) \frac{n}{N} \frac{n-1}{N-1}=4 \frac{6}{20}=\frac{6}{5}
$$

The above calculations, basing on the proposition (2), on are the same with the calculations driving to the relation (1.1) in Section (1.4) of the first published article A, and relative to the previous (3) here we have:

$$
\xi_{M 0}=P(v+1 \| v) / P(v)=(2 / 4) /(3 / 5)=5 / 6<1 .
$$

And general is valid:

$$
P(v)=n / N>(n-1) /(N-1) \Leftrightarrow n<N .
$$

Which condition obviously is in general case correct that is for the above relation (3):

$$
\xi_{M 0}=P(v+2 \| v) / P(v)=[(n-1) /(N-1)] /[n / N]<1
$$

So the factor $\xi_{M 0}$ with the condition $n \ll N$ gives:

$$
\lim _{N \rightarrow \infty} \xi_{M 0}=1-(1 / n)
$$

The (5) applies in RSI $\delta_{M}$ where $N=q_{M+1}^{2}-q_{M}^{2}$ and $n$ is the number of primes inside this random silver interval (RSI) with $q_{M}=\max \left(Y_{M}\right)$ where from the 1 of "Nomenclatures" (section in Appendix at the end of this article B) is $Y_{M}=\left\{2,3,5, \cdots, q_{M}\right\}$. Now we can easily estimate the limit of the above defined "arithmetical length" $N=d_{M}$ of RSI $\delta_{M}$ as the index $M$ tends to the infinite. From the inequality (4.5), mentioned in 10 of "Nomenclatures" (section in Appendix), and from the fact that the number of prime numbers is infinite, that is $\lim _{M \rightarrow \infty}\left(q_{M}\right)=\infty$, we easily find that the limit of the length $N=d_{M}$ of the RSIs is also infinite. Really:

$$
d_{M}>4 q_{M} \Rightarrow \lim _{M \rightarrow \infty}\left(d_{M}\right)>\lim _{M \rightarrow \infty}\left(4 q_{M}\right)=\infty \Rightarrow \lim _{M \rightarrow \infty}\left(d_{M}\right)=\infty
$$

Let organizes all the formulas here. According to the definitions in 5 of "Nomenclatures" (section in Appendix at the end) and from the calculations of the previous example of the eggs we can easily prove the below relations for any RSI $\delta_{M}:$

$$
\begin{gathered}
P(v)=P_{M 0}=\frac{n}{N}=\frac{N_{M 0}}{d_{M}} \\
n_{t}=N_{M 0, t w i n}=\frac{N_{\text {total twies }}}{N_{\text {total cases }}}=\frac{(n-1) n}{N}=\left(N_{M 0}-1\right) P_{M 0} \\
n_{t}=N_{M 0, t w i n}=(N-1) \xi_{M 0}\left(P_{M 0}\right)^{2}=n(n-1) / N \\
\xi_{M 0}=\frac{n-1}{N-1} \frac{N}{n}=\frac{\left(N_{M 0}-1\right) d_{M}}{\left(d_{M}-1\right) N_{M 0}}
\end{gathered}
$$

From the (9) we confirmed that:

$$
\text { If } n \in\{0,1\} \Rightarrow n_{t}=N_{M 0, t w i n}=0
$$


If $2 \leq n=N_{M 0}<N=d_{M} \Rightarrow 1 / 2<\xi_{M 0}<1$

We can do many tests for the above relations with different examples by different values $N, n$.

We will also give a result that we proved, but indifferent to the proof of the twins' conjecture here. In an Euclidean (flat) space of dimensions $k$ we consider a $k$-dimensional parallelepiped with lengths of its sides: $N_{1}, N_{2}, \cdots, N_{k}$ which are natural numbers. Let define now $N=N_{1} N_{2} \cdots N_{k}, W=\sum N_{i(1)} N_{i(2)} \cdots N_{i(k-1)}$ evidently with the all possible combinations and with indexes $i(m) \in\{1,2, \cdots, k\}$ different per two. If we randomly place $n$ eggs (representing each of them the event 1 ) in the $N$ positions (the empty positions evidently correspond to event 0 ) then the corresponding result of the relation (9) for the on average expected twins will be:

$$
n_{t}=(n / N)[(n-1) /(N-1)](k N-W)
$$

This result may be related with entropy's problems in topology etc.

Proof:

Let's start with a space $k=2$ dimensions. Let be a rectangle with two sides with lengths $N_{1}=2, N_{2}=3$. This includes 6 square cells with $x=7$ inner boundaries between them, while the (outer) perimeter has $\lambda=2\left(N_{1}+N_{2}\right)=10$ outer boundaries of the cells. We first want to find the number $x$ of the inner boundaries computationally. Multiplying the total number $N=N_{1} N_{2}$ of the square cells by the number $2 k$ of the boundaries of the perimeter of each square cell of these we find $\lambda+2 x$, because each inner boundary of cells was calculated twice while each outer boundary of cells was calculated once. Therefore we conclude that $2 k N=\lambda+2 x$, and next expanding this result we get $x=k N-\left(N_{1}+N_{2}\right)=2 \times 6-(2+3)=7$ internal boundaries. And for the proof of the above relation (14) we can obviously write $n_{t}=(n / N)[(n-1) /(N-1)] x$. That is, for two dimensions $x=k N-W$. We can easily generalize for more dimensions bearing in mind that on the outer surface $k$-1-dimensions of a $k$-dimensional cube [defining by arbitrary multitude (number) of dimensions] reside $N(k, m)=k_{(m)} 2^{k-m} m$-dimensional "cubes" with $m<k$. For example on the 2-dimensional surface of a typical three-dimensional cube there are: $N(3,2)=3_{(2)} 2^{3-2}=3 \times 2=6 \quad$ faces, $\quad N(3,1)=3_{(1)} 2^{3-1}=3 \times 4=12 \quad$ edges and $N(3,0)=3_{(0)} 2^{3-0}=1 \times 8=8$ vertices. And for the number of sides of a square $N(2,1)=2_{(1)} 2^{2-1}=2 \times 2=4$ etc. We can also see that the quotient of the perimeter $D$ of a geometric shape of the plane to the area $A$ of its surface corresponds to a magnitude $S$ associated with the entropy of the geometric shape. That is $S=D / A$. So if we calculate $S$ for a circle, for a square, for a rhombus etc which have the same area $A$ we will find that the circle has smaller $S$ than the square one that has less $S$ than the rectangle and so on. That is, the smaller $S$ has a geometric shape, the more axes and centers of symmetry it has. However, if we divide it into cells, we can calculate its entropy from known methods and formulas and then we can correlate this entropy with S. We can then study these correlations in spaces (manifolds) of larger dimensions. So we can find methods 
to calculate [taking as well in account the previous relation of Shannon] the bits of "average information" (or entropy) of a geometrical object in Euclidean and as well in curved manifolds and with arbitrary number of dimensions which as well corresponds to events.

A3) We will prove one more proposition: Let be two arrangement sets $A, B$ of positive real (for example rational) numbers:

$$
A=\left\{a_{1}, a_{2}, \cdots, a_{N}\right\}, \quad B=\left\{b_{1}, b_{2}, \cdots, b_{N}\right\}
$$

and also let the product symbols:

$$
\begin{gathered}
X_{n}=a_{n} X_{n-1}=a_{n}\left(a_{1} a_{2} \cdots a_{n-2} a_{n-1}\right), \quad X_{1}=a_{1}, n>1 \\
Y_{n}=b_{n} Y_{n-1}=b_{n}\left(b_{1} b_{2} \cdots b_{n-2} b_{n-1}\right), \quad Y_{1}=b_{1}, n>1
\end{gathered}
$$

This permits us to make any permutation between the elements of the set $A$ simultaneously with the homologous elements of set $B$. The rearrangement obviously is absolutely arbitrary. We can prove that if is valid $a_{i} a_{j}>b_{i} b_{j}$ for every pair $i, j \in\{1,2, \cdots, N\}$ with $i \neq j$, then will be valid:

$$
X_{n}>Y_{n}, \forall n=2,3, \cdots, N \text {. Proposition: }
$$

Proof: The above proposition (15) for index $n=2$ is valid from our hypothesis. Let hypothesized that the relation (15) is valid for $k \geq 2$ :

$$
a_{k} X_{k-1}>b_{k} Y_{k-1}
$$

As we know from the method of perfect mathematical induction, it suffices to show the relation (15) for $n=k+1$. So, "let we assume the relation (15) to be valid for $n=k$, as we assumed just now, and let we assume simultaneously that the relation (15) not apply to $n=k+1$ ". Let be this Proposition (17).

Now, according to the other mathematical method of "negation introduction" (reduction ad absurdum) it is enough to show that the last proposition (17) leads to an absurd result. Really, if is valid the (17) we conclude that must exist two numbers $a_{k+1}, b_{k+1}$ [except of these in (16)] so we have:

$$
a_{k+1} a_{k} X_{k-1}<b_{k+1} b_{k} Y_{k-1}
$$

But the standard hypothesis (16) can use as well these previous factors $a_{k+1}, b_{k+1}$ indifferent to according on (16) what these two previous factors are, because obviously the relation (16) always applies to any two factors:

$$
a_{k+1} X_{k-1}>b_{k+1} Y_{k-1}
$$

Multiplying the members of the relations (16), (19) we get:

$$
a_{k} a_{k+1} X_{k-1}>b_{k} b_{k+1} Y_{k-1}
$$

We thus arrived to an absurd result because the (20) is the refusal of (18). So the relation (17) is not valid and the proof of (15) has been completed. We could start from $a_{i} a_{j}>b_{i} b_{j}$ and similarly to the above proof we can try to prove the (15) initially for only three numbers to make clear the picture of this proof. That is, if the proposition is not true then must existing two numbers $a_{m}, b_{m}$ so to be $a_{i} a_{j} a_{m}<b_{i} b_{j} b_{m}$ from which we again conclude that $a_{m}<b_{m}$ 
and then $a_{i} a_{j}\left(a_{m}\right)^{2}<b_{i} b_{j}\left(b_{m}\right)^{2}$. But from the other hand by initial hypothesis we have that $a_{i} a_{m}>b_{i} b_{m}, a_{j} a_{m}>b_{j} b_{m}$ and multiplying the members of the two relations we get the refusal of the previous relation: $a_{i} a_{j}\left(a_{m}\right)^{2}<b_{i} b_{j}\left(b_{m}\right)^{2}$ and so on.

A4) If we replace the sets $A, B$ of numbers by new sets $A^{*}, B^{*}$ of events $a_{i}^{*}, b_{i}^{*}$ and then if is valid that $P\left(a_{i}^{*}\right.$ or $\left.a_{j}^{*}\right)>P\left(b_{i}^{*}\right.$ or $\left.b_{j}^{*}\right)$ for every pair $i, j \in\{1,2, \cdots, N\}$ with $i \neq j$ we conclude that will be valid that:

$$
P\left(a_{i 1}^{*} \text { or } a_{i 2}^{*} \text { or } \cdots \text { or } a_{i m}^{*}\right)>P\left(b_{i 1}^{*} \text { or } b_{i 2}^{*} \text { or } \cdots \text { or } b_{i m}^{*}\right)
$$

To prove the (21) we can use the (83) of Section 4 below, but with probabilities of our hypothesis instead the fractions there, and similarly to the proof there by using the (15) we can correlate the two expansions of (81) with probabilities for the $A^{*}, B^{*}$ instead the fractions. Taking into account the known probability theory for many alternatively events we can complete this proof. Next, we can try to formulate a corresponding theorem between two classes $A^{* *}, B^{* *}$ with many intersected each other sets, by multitude $\mathrm{N}$ for every class, proving this theorem between the common subsets (of intersections) on the basis of the previous analysis, because the theory of probability is connected by the theory of sets.

\section{B) Connection by the first article $\mathrm{A}$}

According to the definitions we said that the symbol $q_{v}$ (with $v \in N$ ) symbolize the prime numbers: $q_{0}=1, q_{1}=2, q_{2}=3, q_{3}=5, \cdots$

We will define now some categories of silver intervals and then bellow in Section 2 we will give arithmetical examples as well for these categories as for their interior:

The interval which is presented at Figure 1, in Section 4 of first article A that we represented above again, we will call "Real Silver Interval" (RSI) if, and only if, it represents the real distribution of bands in a real (true) silver interval. In Section 3 of first article A we defined the band of $q_{s}$-multiples [prime-multiples of some silver interval $\left.\delta_{M}=\left[q_{M}^{2}, q_{M+1}^{2}\right)\right]$ the subset interval $b_{s}(M)=\left[\theta_{s}(1, M), \theta_{s}(\tau, M)\right]$ that includes only natural numbers between the positions of first " 1 " and last " $\tau$ " multiples of corresponding prime number $q_{s}$ in the same silver interval. In Section 3 of A also we gave the necessary definitions and examples relative to bands. Two bands in the same real silver interval

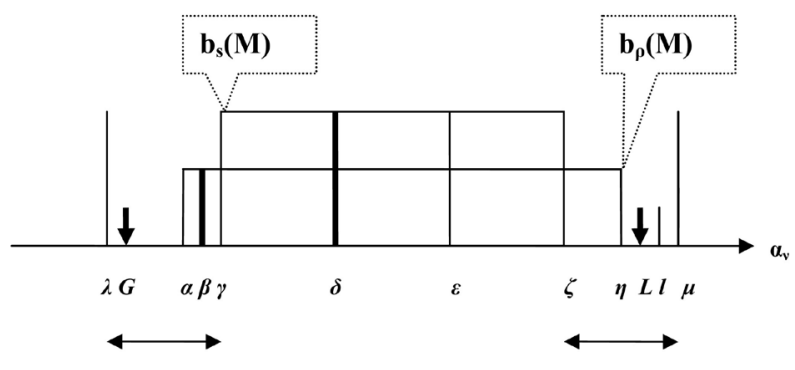

$\mathrm{q}_{\mathrm{s}}$

$\mathrm{q}_{\mathrm{s}}$

Figure 1. Silver interval. 
(RSI) $\delta_{M}$, and in relative to their limits, are related only in two ways. One way is to be included the one band inside the other band as it is in the case of bands $b_{s}(M)=[\gamma, \zeta], b_{\rho}(M)=[\alpha, \eta]$ in Figure 1 of Section 4 in first article A. We will call this way as "not intersection way". The other way is these bands intersect each other and we will call this second way as "intersection way". The distribution way where the beginning of second band $B 2$ appears after the end of first band $B 1$ (in the same real silver interval) does not take place. The reason is that in this case the left distance $L 1$ between the left border of $B 2$ and the left border of RSI $\delta_{M}$ must satisfy the inequality $L(B 2)>L 1>L(B 1)$ and similarly the right distance $L 2$ between the right border of B1 and the right border of $\delta_{M}$ must satisfy the inequality $L(B 1)>L 2>L(B 2)$, where $L(B 1), L(B 2)$ represent the two lengths of bands $B 1, B 2$, and adding these two double inequalities we result in irrational conclusion.

Next we'll define as "Virtual Silver Interval" (VSI) a silver interval where every band of $\delta_{M}$ is expanding theoretically from one border (boundary) of $\delta_{M}$ to another border of $\delta_{M}$, so all bands overlap theoretically each other perfectly and moreover in this manner their erasers can interact perfectly as the theory drives to (4.7) of article A predicts. To understand it we must define the "erasers".

In Section 4 of article A (and after Figure 1) we defined as "eraser" every multiple of a prime number $q_{s}$ at a place $v$ (in RSI $\delta_{M}$ or in VSI) because every such eraser deletes the possibility to be the integer $v$ prime number. These erasers initially are "real erasers" in RSI. But in calculations using the relation (4.7) these erasers [which as we said correspond to prime numbers of $Y_{M}=\left\{q_{1}, q_{2}, q_{3}, \cdots, q_{M}\right\}$ which has been defined at the beginning of Section 2] interact in a VSI each other perfectly and in absolutely accordance with the results of these relations, because the erasers distributed perfectly in total interval $\delta_{M}$. But absolutely the same interaction does not happen in RSI (where again the erasers coincident only with the multiples of primes $q_{s}$ ) because the bands have not now a complete overlap as it was happening just before in virtual silver interval (VSI). Every band $b_{s}(M)$ is a set of eraser $q_{s}$-multiples of $Y_{M}$ and therefore the overlap of two bands $b_{s}(M), b_{n}(M)$ causes a partial overlap between these sets of two kinds of erasers $\left\{q_{s}\right.$-multiples, $q_{n}$-multiples $\}$ that is a partial coincidences of them on some points (natural numbers of silver interval) only inside overlapping intervals of these two bands. The statistics that is expressed by the relations (2.1) or (2.7) of article A work perfectly only for VSI, because the bands only in the VSI are expanding into all its length (which is the same length with the length of its corresponding RSI). This condition is necessary for the correctness of results of (2.1) or (2.7). We can thus expand and study the coincidences of the erasers of different kinds of multiples between three or more bands define common intervals of overlap in all length of VSI or in part of length in the RSI. We define from now on as "real clear deletion multitude" inside an interval the multitude of essential (clear) deletions from all kinds different erasers in this interval by rule to counting as one deletion all together the 
degenerate deletions (from all the different kind of erasers) when these are coincident on a point (on a natural number inside this silver interval). And similarly we will define the "virtual clear eraser multitude" for virtual silver interval, minded that in VSI the above definition has no specific points of coincidences. The general rule is: "The greater the clear density of the deletions, the fewer the prime numbers". We can also use the dynamic term "moving eraser" for deletions of specific kind $q_{s}$-multiples for its many deletions in an interval inside a silver interval RSI or VSI so to have a moving image of this specific eraser's deletions.

As we analyzed in Section 4 of first article A, and after Figure 1, this arithmetical phenomenon decreases the clear multitude of virtual deletions (from all kinds of erasers) in VSI, because the deletions partially and virtually coincident more times each other on non specific points (natural numbers) inside VSI, in comparison to the real deletions (from all kinds of erasers) which coincident partially again each other but now (in contrary to VSI case) on specific points (of natural numbers) inside the corresponding RSI. An important phenomenon of partial degeneration that increases the "real clear deletion multitude" and therefore increases the "real clear density of deletion" of erasers relative to "virtual clear deletion multitude" and "virtual clear density of deletion" of virtual default case. So we define as "clear density" the one that ultimately determines the number of prime numbers. And so we need define a virtual silver interval " $g$ " with virtual clear density of deletion greater than $1 / q_{s}$, that is a "special virtual deletion density": $\rho_{s}>1 / q_{s}$, which in the article A we called "true density" of calculations for relation (4.16). The density $\bar{\rho}_{s}=1 / q_{s}$ in (4.7) for the default virtual silver interval we called "active density" and in calculations obviously this density drives to a greater default virtual multitude of primes in comparison to the real prime's multitude in real $\delta_{M}$. To avoid confusion we will henceforth adopt only the term "virtual clear density of deletion" or simply "virtual density" $\rho_{s}=g / q_{s}$ because in calculations we always use a virtual silver interval and so every density refers to some virtual silver interval. The factor $g$ (of choice of the "virtual silver interval $g$ ") gives the virtual modification for the density increment of virtual deletions in "virtual silver interval $g$ " and takes values equal or greater than 1 . The case equal to 1 refers to the default virtual silver interval of RSI $\delta_{M}$. At this point we must make clear that the cases where the "virtual clear deletion multitude" $Y$ l of virtual erasers is smaller than "real clear deletion multitude" $Y$ (in the corresponding real silver interval) do not interest us, because in calculations we need a virtual silver interval " $g$ " with smaller multitude $X 1$ of primes in it than the real multitude $X$, so if we prove that the $X 1$ is infinite, then automatically we will have as final conclusion that the real multitude $X$ is infinite also. Consequently we must choice $g>1$ and below we will prove that the case of every "special virtual silver interval" with $g=2$ (which we used for our calculations in article A) really corresponds to a multitude $X 1$ of primes smaller than real multitude $X$ of corresponding RSI, and so our calculations are really correct. 
Then, in addition we'll prove here that all cases with $g>1.3$ correspond to $X 1<X$ also. Obviously the proof in article was depended on case $g=2$ [relation (4.17)]. But as we refer [in (3) of section “The steps"], in the next Section 4 the proof of this point (that declares that case $g=2$ corresponds to $X 1<X$ and so is valid for our realized statistical calculation) now will be different. The reason is that this point in first published article A arose with introduction "two erasers in every band", hoping there we can arrive to a virtual silver interval " $g$ " with more deletions than the case of real silver interval. But we owe a clear proof for (4.17). So finally we will understand the critical point of total proof more easily and absolutely clearly, so we will are sure that our choice of case $g=2$ really corresponds to relation $X 1<X$, and so in the Section 5 with new techniques we will make the proof perfect.

\section{Band's Theory in Silver Intervals}

We defined before, that every Real Silver Interval (RSI) in $N$ :

$$
\delta_{M}=\left[q_{M}^{2}, q_{M+1}^{2}\right)
$$

$M \in N$ with unknown real density $\rho_{s}^{*}>\bar{\rho}_{s}=1 / q_{s}$ for every prime number $q_{s} \in Y_{M}=\left\{q_{1}, q_{2}, q_{3}, \cdots, q_{M}\right\}$ corresponds to a set including an infinite multitude from Virtual Silver Intervals (VSI) with densities $\rho_{s}=g / q_{s}$ for every VSI. The above value $\rho_{s}=\bar{\rho}_{s}$ would be in the factors of relation (4.16) of article A, only if we choose $g=1$ ]. In article A we are interested only for $g$ values with the property $1 \leq g \leq 2$. The role of the parameter $g$ will be analyzed just below, e.g. see below relation (24). The dash above the symbols $\mathrm{P}$ etc, in relations (2.1) or (2.7) of article A, indicates that the corresponding probabilities and densities refer to the default VSI that we defined by choice $g=1$. In other words the VSI $g=1$ corresponds to that we called before default virtual interval. Another VSI defined by choice $g=2$ we will call proper virtual interval. We can understand the VSI for some $g$ easily. For example let we choose the RSI:

$$
\delta_{4}=\left[q_{4}^{2}, q_{4+1}^{2}\right)=\left[7^{2}, 11^{2}\right)=[49,121)
$$

We define two types of lengths in $\delta_{M}$ between two places of natural numbers $m, n$ inside $\delta_{M}:$ A) The type of arithmetical length $l([m, n])=n-m+1$ that counts the natural numbers of the interval $[m, n]$. B) The geometrical length $L([m, n])=n-m$ that counts the units (the multitude of 1 ) included in $[m, n]$. For example, the relations (4.4), (4.5) at the beginning of section 4 , in the first article A, define the arithmetical length of a band and of a silver interval, whereas the relation (4.6) there represents the geometrical length $L$ of the interval $\left[0, q_{M+1}^{2}-1\right]$ on the known axis $N$. The relation between arithmetical and geometrical length of the same interval $[m, n]$ evidently is $l([m, n])=1+L([m, n])$, and the (4.3) defines the arithmetical length $d_{M}=q_{M+1}^{2}-q_{M}^{2}$ of silver interval $\delta_{M}$. C) We must point that the product $p l$ of arithmetical length $l$ inside $\delta_{M}$ with a probability $p$ of an event $e$, on every natural number of the interval $l$, gives the fraction (per unit, whereas per cent is $100 \times l p \%$ ) of this event $e$ on the total 
numbers of the arithmetical interval $l$, if we considerer that $p d_{M}=1$. This event $e$ could be e.g. a kind of deletion of some eraser (or in other case of many erasers together) inside the silver interval $\delta_{M}$ with probability $p$ on every natural number of the interval $l$.

Let be the above A, B, C definitions as the Proposition: (22)

Let be the previous example of $\delta_{4}$, with its sub-sequence $Y_{4}=\{2,3,5,7\}$. We defined $Y_{M}$ at the beginning of Section 2 of the article A. This RSI $\delta_{4}$ corresponds to a collection of the VSIs from different values of $g$ that we will symbolize and we will define as follows:

$$
\delta_{4}^{v i r}(g)=\left[q_{4}^{2}, q_{4+1}^{2}\right)=[49,121)
$$

by the factor:

$$
g=\mathrm{K} / \Lambda
$$

The numbers K, $\Lambda$ are prime numbers to each other, so to be the number $g$ a positive rational number. The symbol "vir" means virtual (silver interval). The arithmetical length of VSI $\delta_{4}^{\text {vir }}(g)=[49,121)$ according to its previous definition obviously is $l_{M}(g)=121-49=72$. Accordingly to the relation (4.16) we will define as probability to find a prime in $\delta_{4}^{\text {vir }}(g)$ the next expression by $1 \leq g<2$ :

$$
P_{M}(g)=\prod_{j=1}^{M_{v}} F_{j}(g)=\left(1-\frac{g}{2}\right)\left(1-\frac{g}{3}\right)\left(1-\frac{g}{5}\right)\left(1-\frac{g}{7}\right) \cdots\left(1-\frac{g}{q_{M v}}\right)
$$

And choosing $g=1$ for default VSI we calculate:

$$
P_{4}(1)=\left(1-\frac{1}{2}\right)\left(1-\frac{1}{3}\right)\left(1-\frac{1}{5}\right)\left(1-\frac{1}{7}\right)=\frac{24}{105}
$$

This result represents the fraction of prime numbers in $\delta_{4}^{\text {vir }}(1)$ and therefore it predicts a multitude $n=(121-49)(24 / 105)=1728 / 105 \cong 16.4$ of prime numbers which is a not correct result because in this RSI are included 15 prime numbers as Table 1 of article A shows. Thus the rational number 1728/105

Table 1. Prime multitude in silver intervals.

\begin{tabular}{ccc}
\hline Real Silver Intervals (RSIs) & $\begin{array}{c}\text { True Multitude } \\
\text { of prime numbers }\end{array}$ & $\begin{array}{c}\text { Expected multitude } \\
\text { of prime numbers in VSIs g }=1\end{array}$ \\
\hline$[4,8]$ & 2 & 2 \\
{$[9,24]$} & 5 & 5 \\
{$[25,48]$} & 6 & 6 \\
{$[49,120]$} & 15 & 16 \\
{$[121,168]$} & 9 & 9 \\
{$[169,288]$} & 22 & 23 \\
{$[289,360]$} & 11 & 12 \\
{$[361,528]$} & 27 & 28 \\
{$[529,840]$} & 47 & 51 \\
{$[841,960]$} & 16 & 18 \\
{$[961,1368]$} & 57 & 62 \\
{$[1369,1680]$} & 44 & 46 \\
\hline
\end{tabular}


represents the virtual multitude of primes for default VSI $g=1$. This number could be useful for our calculation but as we explained in the first article (A) it is greater than the real multitude because the bands in the RSI $\delta_{4}=[49,121)$ do not have a complete overlap as it is happening in the VSI $\delta_{4}^{\text {vir }}(1)$ and so the deletions in RSI are distributed in a greater (of one dimension) space with a less overlap to each other than the above relation (25) predicts. In other words the "virtual clear deletion multitude" (that we defined in Introduction here) decreases in comparison to the corresponding "real clear deletion multitude" (that we also defined in Introduction) of RSI $\delta_{4}=[49,121)$ because they in default VSI (defined as we said by choice $g=1$ ) have the theoretical deletion coincidences of (4.7) of A article in contrary to the deletions of its corresponding RSI where for "real clear deletion multitude" in general case (random choice) we have no correct general relation to calculate this. The problem now is to find a new $g$ greater than 1 for VSI $\delta_{4}^{\text {vir }}(g)$ which will generate the properly great density of deletions so that accordingly to (24) the VSI $\delta_{4}^{\text {vir }}(g)$ would have a properly less virtual multitude of prime numbers than the true multitude 15 , not only especially for this RSI $\delta_{4}=[49,121)$ but in the general case. And therefore this will drive us to calculations with a less multitude of prime numbers than in real exist. Conclusively, if we in this way can prove that the virtual multitude of twins of all together VSI $\delta_{M}^{v i r}(g)$, e.g. with common $g>1$, is infinite, then we will immediately conclude that the true (real) multitude of twins of all together RSI $\delta_{M}$ in $N$ is infinite also.

Finally, we will give some examples with bands and their deletions using the real silver interval RSI $\delta_{4}=\left[q_{4}^{2}, q_{5}^{2}\right)=\left[7^{2}, 11^{2}\right)=[49,121)$ :

This RSI includes four bands with their corresponding moving erasers:

1) $b_{2}(4)=\left[\theta_{2}(1,4), \theta_{2}(\tau, 4)\right]=[50,120]$, with 36 deleted numbers in it from moving eraser $q_{2}$-multiple.

2) $b_{3}(4)=\left[\theta_{3}(1,4), \theta_{3}(\tau, 4)\right]=[51,120]$, with 24 deleted numbers in it from its moving eraser $q_{3}$-multiple.

3) $b_{5}(4)=\left[\theta_{5}(1,4), \theta_{5}(\tau, 4)\right]=[50,120]$, with 15 deleted numbers in it from its moving eraser $q_{5}$-multiple.

4) $b_{7}(4)=\left[\theta_{7}(1,4), \theta_{7}(\tau, 4)\right]=[49,119]$, with 11 deleted numbers in it from its moving eraser $q_{7}$-multiple. Also in RSI $\delta_{4}$ the "real clear deletion multitude" is equal to $72-15=57$, accordingly to the definition of "real clear deletion multitude" in Introduction before. And also to the definition "real clear density of deletion", in Introduction before, for our RSI we can put:

$$
\rho^{*}=57 / 72 \cong 0.791
$$

We will distinguish the "clear deletion density" from the previous density $\rho_{s}=g / q_{s}$ that we referred at the beginning of this Section 2. The "clear deletion density" will be calculated below in relation (28) and is different from $\rho_{s}$. Obviously the first band $b_{2}(4)$ coincides with the third band $b_{5}(4)$, while apart from this pair all the other 5 pairs (which are formed from these 4 bands) 
don't coincide. There are also natural numbers (points) in RSI $\delta_{4}$ where the deletions from two or more moving different erasers coincide, e.g. in position 50 (which is the second position of RSI $\delta_{4}$ ) where the deletions from moving erasers $q_{2}$-multiple, $q_{5}$-multiple coincide and the point 56 where the same take place by the pair $q_{2}$-multiple, $q_{7}$-multiple etc.

For VSI $\delta_{M}^{\text {vir }}(g)$ we must emphasize that it is an ideal interval that has as its archetype the RSI $\delta_{M}$. We can imagine that $\delta_{M}^{v i r}(g)$ is placed as a slide on $\delta_{M}$ where it is applied perfectly and constantly compares its data with $\delta_{M}$. Deletions in $\delta_{M}^{v i r}(g)$ are always ideal and fairly distributed in it based on the relation (24). We can thus imagine any virtual eraser $q_{s}$-multiple of $\delta_{M}^{\text {vir }}(g)$ as an arrow that has probability of $g / q_{s}$ hitting any natural number inside the VSI $\delta_{M}^{\text {vir }}(g)$. Therefore the probability of being prime number on every position $v$ of VSI $\delta_{M}^{\text {vir }}(g)$ will be constant equal to $P_{M}(g)$ and will be given by (24), while according to (1.1) in Section 1 of the first article A for the corresponding twin, in the same random position, this probability was equal to $\left[P_{M}(g)\right]^{2}$, but as we said below we replace it by (3).

Let us now compare the relations (24) and (25). Selecting e.g. the value $g=1.43$ (which is greater than 1.3) these relations give us respectively $P_{4}(1.43) \cong 0.085$ and $P_{4}(1) \cong 0.228=16.45 / 72$. We observe the drastic reduction of the prime number multitude in VSI $\delta_{4}^{\text {vir }}(1.43)$ in relation to RSI $\delta_{4}$ (where we saw that $P=15 / 72$ ) because it is $g=1.43>1.3>1$. We thus manage to already have one $g=1.43$ which promises that the proof we are looking for is feasible. In other words, although the default VSI $\delta_{4}^{\text {vir }}(1)$ has a larger multitude of prime numbers (16) than the RSI $\delta_{4}$ that has 15 (something that is true for intervals in Table 1 of article A) now we managed to reverse the situation by replacing the default case of VSI of $g=1$ with a new VSI of $g=1.43$. So it suffices to prove that 1 ) this is true generally for VSI $\delta_{M}^{\text {vir }}(g)$ with $2 \geq g>1.3$ and 2) the set of proper VSI $\delta_{M}^{\text {vir }}(g)$ includes an infinite multitude of twins, as we analyzed before. This we already did in article A and now, as we said, we will give bellow in Section 4 a new proof that clarify the critical point of case (1) just before. The " $y$ " - " $g$ " diagrams are also interesting, choosing for example two cases from them with $y=P_{M}(g), \quad y=P_{M}(g) / P_{M}(1)$. These diagrams hide information about distribution of prime numbers in silver intervals with $g \in Q \subset R$.

Developing the above relation (24) we turn the product into a sum, and so we get:

$$
P_{M}(g)=1-\left[\sum_{s=1}^{s=M} \frac{g}{q_{i}}-\sum_{I_{1}=1}^{I_{1}=M} \sum_{I_{1} \neq I_{2}=1}^{I_{2}=M} \frac{g}{q_{I_{1}}} \frac{g}{q_{I_{2}}}+\cdots+(-1)^{N+1} \sum_{I_{1}=1}^{I_{1}=M} \cdots \sum_{I_{k} \neq I_{N}=1}^{I_{N}=M} \frac{g}{q_{I_{1}}} \cdots \frac{g}{q_{I_{N}}}\right]
$$

Where the proposition $I_{k} \neq I_{N}$ means that the indexes in products in every sym are different between them. And for the new greater virtual density, putting $\rho_{s}=g / q_{s}$, we will have the corresponding smaller prime numbers probability of VSI $g>1$ (relative to the default VSI $g=1$ probability): 


$$
\begin{aligned}
P_{M}(g)= & -\left(g \sum_{s=1}^{s=M} \rho_{s}\right)+\left(g^{2} \sum_{I_{1}=1}^{I_{1}=M} \sum_{I_{1} \neq I_{2}=1}^{I_{2}=M} \rho_{I_{1}} \rho_{I_{2}}-\cdots\right. \\
& \left.+(-1)^{N} g^{N} \sum_{I_{1}=1}^{I_{1}=M} \cdots \sum_{I_{k} \neq I_{N}=1}^{I_{N}=M} \rho_{I_{1}} \cdots \rho_{I_{N}}\right)
\end{aligned}
$$

Let us putt $R_{M}(g)=\left(g \sum_{s=1}^{s=M} \rho_{s}\right)$ and

$$
K_{M}(g)=\left(g^{2} \sum_{I_{1}=1}^{I_{1}=M} \sum_{I_{1} \neq I_{2}=1}^{I_{2}=M} \rho_{I_{1}} \rho_{I_{2}}-\cdots+(-1)^{N} g^{N} \sum_{I_{1}=1}^{I_{1}=M} \cdots \sum_{I_{k} \neq I_{N}=1}^{I_{N}=M} \rho_{I_{1}} \cdots \rho_{I_{N}}\right)
$$

By these substitutions the above (27) takes the simplest form:

$$
P_{M}(g)=1-\left(R_{M}(g)-K_{M}(g)\right)=1-U_{M}(g)
$$

Thus we define the quantity:

$$
\rho=U_{M}(g)=R_{M}(g)-K_{M}(g)
$$

Let us now develop the above relation (28) for only two random factors $F_{i}, F_{j}$ of (24) setting for them $x=1 / q_{i}$ and $y=1 / q_{j}$. So will have that $R(g)=g(x+y), K(g)=g^{2} x y$. Conclusively, for this simple case we find:

$$
\rho=U(g)=g(x+y)-g^{2} x y
$$

This relation (29) represents the "virtual clear density of deletion" [that we already used in relation (26)] or simply "virtual density" as it is formed by the interaction of the erasers between only two bands $b_{i}(M), b_{j}(M)$ for some values $g, M$. This relation will be useful in bellow Section 4 . The general rule as we said in Introduction here is: "The greater the clear deletion density, the fewer the prime numbers".

According to our definitions before, we calculated in (26) the "real clear density of deletion" $57 / 72$ of the specific RSI $\delta_{4}$. Similarly the quantity $\rho=U(g)$ generally can represent the "virtual clear density of deletion" of VSI. For example for the default VSI $\delta_{4}^{\text {vir }}(1)$ we will have the "virtual clear density of deletion" or simply "virtual density":

$$
\rho=U(4)=1-P_{4}(1)=1-16 / 72=56 / 72 \cong 0.777
$$

This value $56 / 72$ is smaller than the corresponding real $57 / 72$ because the RSI $\delta_{4}$ has "real clear deletion multitude" equal to 57 whereas the default VSI $\delta_{4}^{v i r}(1)$ has "virtual clear deletion multitude" equal to 56.

As we said before, we are now looking for every $\delta_{M}^{v i r}(g)$ that has greater "virtual clear density of deletion" than the "real clear density of deletion" for all values of $M$. For example choosing the previous value $g=1.43>1.3$ and using the relation $(28)$ we find $\rho=1-P_{4}(1.43) \cong 1-0.085=0.915$.

We observe that this value of new VSI $g=1.43\left[\delta_{4}^{\text {vir }}(1.43)\right]$, in contrary to the default $\delta_{4}^{\text {vir }}(1)$ that has $\rho$ about 0.777 , is now significantly greater than the corresponding value $\rho^{*}=57 / 72 \cong 0.791$ of RSI $\delta_{4}$. Thus we looking for any values $g, M$ to be: 


$$
\rho(\text { proper VSI })>\rho(\text { RSI }) \geq \rho(\text { default VSI })
$$

that is the general case of our special example here: $0.915>0.791>0.777$. We'll define as "proper VSI $g$ " every $\delta_{M}^{\text {vir }}(g)$ that for all its infinite natural numbers $M=1,2,3,4, \cdots$ satisfies the inequality:

$$
\rho(\text { proper VSI } g>1)>\rho(\text { RSI }) \geq \rho(\text { default VSI } g=1)
$$

All these because we said that we want to do the proof for the twins using a suitable $\delta_{M}^{\text {vir }}(g)$ that satisfies the relation (30). Because by adding the twins for $M=1,2,3,4, \cdots$ and for a single proper VSI (that is, with a specific value of $g>1$ ) if we manage and prove that these virtual twins of this specific $g>1$ have infinite multitude then we will conclude that the twins of all infinite corresponding RSI on basis of (30) will also have infinite multitude, by the condition that the statistical distribution of the twins within the infinite number of all RSIs is such that we can use the inequalities we prove. We study this problem below. Also in Section 4 we will determine the allowable (only in our need) value of the factor $g$. We will thus prove that the specific value $g=2$, with $g=1$ for the first factor, are proper values for our proof.

\section{The Proposition of Catholic Information in a Random Silver Interval}

Based on the principle that nothing happens in Mathematics without a cause, we will formulate the "known" following proposition:

"Let be a set $A$. We symbolize $a_{i}=R(A)$ a random choice of an element from $A$. Random selection or choice we define the selection of a mental machine from $A$, where from any subset of $A$ with $N$ finite multitude of elements the machine select any time one element from them by $P=1 / N$ constant probability (or frequency). On each random selection will be true all the propositions of definition of the set $A$ ". We can include these propositions in a proper definition set $\operatorname{def}(A)$. The set $\operatorname{def}(A)$ we will call Catholic Information o $A$ and the above general definition we will call here as Proposition: (31).

If the set $A$ has infinite multitude of arranged elements we can say that the $a_{i}=R(A)$ in $A$ represents an element of "infinite in set $A$ ", because the infinite in $A$ is defined by the general or Catholic propositions of set $A$, and nothing more. For example, what is the even number "in the middle of set $N^{\text {"? }}$ ? The answer is "all the propositions which are true for the number's form $2 k$ and nothing more". "Something like correlation between the infinity of the numbers and the world of logical propositions ..."

Therefore, the propositions for defining an event $e$ occurring with probability $P(e)$ on $a_{i}=R(A)$ will constitute a new set $B=\operatorname{def}(e)$ of new propositions that will be true on $A$ and therefore will contribute to the form of a third set $G=\operatorname{def}(A) \cup \operatorname{def}(e)$ which includes the above proposition (31) with the definition of the event $e$. This means that the statistical distribution of $e$ in $A$ will be dictated solely by the set $G$.

We will name this Proposition: (32) 
Moreover the set $G=\operatorname{def}(D) \cup \operatorname{def}($ prime) with (35) below define the most general propositions which absolutely describe the statistics of a random prime number in $N$ or in other words they give the distribution of prime numbers probability in $N$. Let us now symbolize by $q_{s} \mid v$ the proposition "The quotient of the division $m=v: q_{s}$ is a natural number". Also we will define the refusal of perfect division in $N$ as follows:

$$
q_{s}, \text { not } \mid v
$$

Let us also symbolize $\alpha<\beta=$ "the event $\alpha$ of $v \in N$ is independent of $\beta$ of $v$, i.e. $P(\alpha \| \beta)=P(\alpha)$ in $N^{p}$, (see 2 of Nomenclatures section in Appendix at the end of this article $B)$.

In general, we will define two events $\mathrm{a}, \mathrm{b}$ (defined by catholic propositions in a set A) be independent $\alpha<>\beta$ when and only when any proposition which change the probability of the truth of one from the two (that must based exclusively only on catholic information of definitions) does not change the probability of truth of the other event [by use again only catholic information (general propositions) coming exclusively only from their general (catholic) definitions]. Also we will symbolize the dependence as $e_{1}><e_{2}$. As we had referred in Introduction in the article $\mathrm{A}$ in combination by Section 2, the set: $B=\operatorname{def}$ (prime) defined as follows:

$$
\operatorname{def}(\text { prime })=\left\{v \in \delta_{M} \| C P=\left\{q_{s}=R\left(Y_{M}\right), \text { not } \mid v\right\}\right\}
$$

The symbol CP declares a Catholic Proposition by general definition. For example the set $D$ of all RSI $\delta_{M}$ can be defined as follows:

$$
\begin{aligned}
& \operatorname{def}(D)=\left\{\delta_{M}=\left[q_{n}^{2}, q_{n+1}^{2}-1\right] \| q_{n},\right. \\
& q_{n+1} \in N_{q} \| N_{q}=\{v \in N \| C P(v)=\operatorname{def}(\text { prime })\}, \\
& G=\operatorname{def}(D) \cup \operatorname{def}(\text { prime })
\end{aligned}
$$

Let random prime

$$
q_{m}=R\left[\delta_{M}=R(D)\right]
$$

With (36) let be the natural number:

$$
x=q_{m}+2
$$

There are two possibilities:
1) $x \in \delta_{M}$
2) $x \in \delta_{M+1}$

Let us examine at first the main case (38) and next we'll refer to the other (39). Let be now the

$$
C P(\mu, v)=\left\{v, \mu=R\left[\delta_{M}=R(D)\right]\right\}
$$

We will examine the next relation which concerns the independence of not divisibility:

$$
\left[q_{s}=R\left(Y_{M}\right), \text { not } \mid v\right]<>\left[q_{s}, \text { not } \mid \mu\right]
$$


In Introduction of first article A we explained that the multiples of a prime number (e.g. 5) do not change their frequency (e.g. equal to 1/5) when we impose to this number $v$ as restriction to be as well $v$ multiple of another prime number (e.g. 7):

$$
P(v=\text { mul. of } 5 \| v=\text { mul. of } 7)=P(v=\text { mul. of } 5)=1 / 5
$$

And this implies that the same would happen for the refuses of divisions of such events. In other words the last proposition will be valid if we reverse events by substituting where "multiple" with "not multiple" inside (42). As we know, for every pair of two prime numbers $q_{a}, q_{b}$ exist always two natural numbers $m, n$ so for two successive multiples of $q_{a}, q_{b}$ we can write:

$$
m q_{a}=n q_{b}+v,
$$

where is valid

$$
v \leq\left|q_{a}-q_{b}\right|
$$

The catholic information set $\operatorname{def}(A)$ choice the elements of $A$ from a wider set $\Omega$ of its reference. Therefore $\operatorname{def}(A)$ contains exclusively and only all the necessary propositions whose truth is equivalent to the coherence of the elements of set $A$. However, each specific element $a_{i}$ of $A$ also must contain and other propositions which belong exclusively to $a_{i}$ by distinguishing $a_{i}$ from all other elements of $\Omega$. The truth of these last propositions of $a_{i}$ constitutes the distinction of the elements $A$ between them. Thus these propositions with value "true" for the distinction of elements $a_{i}$ must have other sets of reference $\operatorname{def}\left(A_{1}\right), \operatorname{def}\left(A_{2}\right), \cdots$. For example, counting the keys of a computer requires the knowledge of both the $\operatorname{def}(A)$ set of identification of keys of their set $A$ so that we do not count with the keys and the chairs in the room, and also we need and other sets of propositions $\operatorname{def}\left(A_{1}\right), \operatorname{def}\left(A_{2}\right), \cdots$. (now foreign to $A$ ) for distinguish the keys between them, so for example we do not count two keys together as one.

According to sentences (31), (32), any proposition that refers to statistical distributions of events in the infinite number of elements of a set $A$ will be valid when, and only when, this catholic statistical proposition is proved exclusively and only by the use of catholic propositions of choice (and therefore definition) of these events on of $A$. E.g. for the proof of a property all points of a straight segment $O H$ in geometry we must use only the general properties of the definition of $\mathrm{OH}$ getting a random on it. These are catholic true propositions which define generally all the geometrical properties $\mathrm{OH}$.

In $1 \mathrm{~b}$ of Nomenclatures (section in Appendix at the end) we define as Golden Interval (GI):

$$
\Delta_{N}=\left[1, q_{1} q_{2} \cdots q_{N}\right] \text {, with sub sequence } X_{N}=\left\{q_{1}, q_{2}, \cdots, q_{N}\right\}
$$

Also we define there the random selection from a set $A$ under restriction:

$$
a_{i}=R(A \| C I R)
$$

According to (31), (32) every random selection (45) will present exclusively 
and only the general propositions of each event defined on the elements of $A$.

Proposition: (46)

E.g. suppose that on $n$ different faces of an irregular polyhedron we have written $n$ prime numbers $q_{M(i)}, i=1,2, \cdots, v$ different per two and on the other hand we have defined the set $A$ to include only these numbers. Suppose we throw this polyhedron $N$ times using it as irregular dice and then we observe that have came $\mu(i), i=1,2, \cdots, v$ times the $q_{M(i)}, i=1,2, \cdots, v$ correspondingly. Let be we now choose numbers with $N$ random choices from the set $A$ where we will have now respectively $m(i), i=1,2, \cdots, v$ for $q_{M(i)}, i=1,2, \cdots, v$. Consequently, according to the above proposition (46), if the above result is the average of infinite number of repetitions, will be valid the next Catholic Proposition $(\mathrm{CI})$ :

$$
\prod_{i=1}^{i=N}\left[q_{M(i)}\right]^{\mu(i)}=\prod_{i=1}^{i=N} q_{M(i)}^{\mu(i)} \neq \prod_{i=1}^{i=N} q_{M(i)}^{m(i)}
$$

The reason of the above inequality is that the polyhedron does not choose the prime numbers randomly, that is with the same probabilities because this is irregular.

According to the above (44) let be $D_{N}=\left\{\delta_{0}, \delta_{1}, \delta_{3}, \cdots, \delta_{K(N)}\right\}$ the set with the all RSIs which are including in GI $\Delta_{N}$, and let be

$\Omega_{N}=q_{1} q_{2} \cdots q_{N}=2 \times 3 \times 5 \times \cdots \times q_{N}$ the arithmetical length of $\Delta_{N}$. We have defined $\delta_{0}=\left[2,2^{2}\right), d_{0}=2^{2}-2, \delta_{1}=\left[2^{2}, 3^{2}\right), d_{1}=3^{2}-2^{2}, \cdots$. Next, we will define:

$$
d_{\Delta(N)}=\Omega_{N}-\left[d_{0}+d_{1}+d_{2}+\cdots+d_{K(N)}\right]-1=\Omega_{N}-q_{K(N)+1}^{2}+1
$$

For example the remaining part of $\Delta_{3}$, that is left over and belongs to the last $\delta_{3}$ which is not completed in GI $\Delta_{3}=\{1,2, \cdots, 30\}=[1,30]$, will have according to (48) the numerical length:

$$
d_{\Delta(3)}=\Omega_{3}-\left[d_{0}+d_{1}+d_{2}\right]-1=\Omega_{3}-q_{2+1}^{2}+1=(2 \times 3 \times 5)-5^{2}+1=6
$$

Next we will prove that:

$$
\lim _{N \rightarrow \infty}\left[d_{\Delta(N)} / \Omega_{N}\right]=0
$$

We know the theorem of prime numbers:

$$
\mathrm{d} n=\mathrm{d} x / \ln (x), x \in N
$$

We always can choose $\delta x / x \rightarrow \mathrm{d} x / x \ll 1$ with any desired accuracy:

$$
\ln (x) / x=(\delta x / x)<\varepsilon, \forall x>X(\varepsilon) \in Q^{+}
$$

Because the sequence $\ln (x) / x$ is descending as $x$ is increasing we can always find $X(\varepsilon)$ for any $\varepsilon>0$. The reason for these is that from (50) the distance between two successive prime numbers can be found putting:

$$
\delta n=1=\delta x / \ln (x) \Rightarrow \delta x=\ln (x)
$$

Putting $X(\varepsilon)=\mathrm{e}^{T}, \varepsilon=\mathrm{e}^{-E}$ from (51) we will have $T-\ln (T)>E$, and so for this is sufficient to select: 


$$
T_{\min }=1+E+\ln (E)
$$

For example if we demand an accuracy $\varepsilon=\mathrm{e}^{-50}$ we will have:

$$
\ln (x) / x=\delta x / x<\mathrm{e}^{-50}, \forall x>X\left(\mathrm{e}^{-50}\right)=\mathrm{e}^{50+5}=\mathrm{e}^{55} .
$$

Therefore according to the previous $\forall \Delta_{N}, \forall \varepsilon>0 \Rightarrow \exists X(\varepsilon) \in Q^{+}$so the distance between two successive RSIs can be used as follows:

$$
\delta\left[d_{M}\right]=q_{M+1}^{2}-q_{M}^{2}=\left(q_{M}+q_{M+1}\right)\left(q_{M+1}-q_{M}\right)=\left(2 q_{M}+\Delta q_{M}\right) \Delta q_{M}
$$

or

$$
\begin{gathered}
\delta\left[d_{M}\right]=(2 x+\delta x)(\delta x)=2 x \ln (x)+[\ln (x)]^{2}, \quad \forall x>X(\varepsilon) \\
\Omega_{N}=\left[q_{1} q_{2} \cdots q_{n(\varepsilon)-1}\right] q_{n(\varepsilon)} \cdots q_{N}=\Pi_{\varepsilon} \prod_{n=n(\varepsilon)}^{n=N}(x+n \delta x), \quad \forall x>X(\varepsilon)
\end{gathered}
$$

And so

$$
\mathrm{d}\left[\Omega_{N}\right]=\Pi_{\varepsilon}(\mathrm{d} x) \sum_{v=n(\varepsilon)}^{v=N}\left[(1+(v / x)) \prod_{v \neq n=n(\varepsilon)}^{n=N}(x+n \ln (x))\right]
$$

According to (52) in the (54), (55), (56) will be:

$$
\mathrm{d} x \leftarrow \delta x=\ln (x),
$$

and so dividing these two last relations we easily get:

$$
\lim _{N \rightarrow \infty}\left[\delta\left(\Omega_{N}\right) / \delta\left(d_{M}\right)\right] \rightarrow \lim _{N \rightarrow \infty}\left[\mathrm{d}\left(\Omega_{N}\right) / \mathrm{d}\left(d_{M}\right)\right]=0
$$

The (57) says that the Gold Interval grows up very faster toward the infinite than the including RSIs in it and therefore we conclude the validity of (49).

For any GI $\Delta_{N}=\left[1, q_{1} q_{2} \cdots q_{N}\right]$ we can define one circle circumference with inscribed regular polygons which we will call golden polygons and will correspond to the prime numbers of the sub sequence $X_{N}=\left\{q_{1}, q_{2}, \cdots, q_{N}\right\}$ of the GI $\Delta_{N}$. We will present them by an example which easily can be generalized for every $N$. Let us get the $\Delta_{3}$. The regular basic polygon of all its inscribed polygons will have (2) (3) (5) $=30$ sides with length 1 . The inscribed polygons will be three and will have numbers of sides $30 / 2=15,30 / 3=10,30 / 5=6$ respectively, corresponding to the prime numbers $2,3,5$. The 2, 3, 5 are the lengths of their sides measured by unit defined by the side of polygon of basis with 30 sides which as we said are all equals to 1 . The common divider of the number of sides between two polygons presents the number of their common vertices. The system of these polygons remains unchanged geometrical construction to their rotations. If we let the $N$ tends to infinite, then the arc corresponding to $d_{\Delta(N)}$ according to (49) will tend to be zero relative to the total circumference which corresponds to $\Delta_{N}$.

Let be now one GI $\Delta_{N}$ with its RSIs $\delta_{0}, \delta_{1}, \delta_{3}, \cdots, \delta_{K(N)}$ inside it.

Ignoring initially these RSIs we can subdivide $\Delta_{N}$ into successive segments $A_{0} A_{1}, A_{1} A_{2}, \cdots, A_{k-1} A_{k}$. It is obvious that there is no way of division that makes the random selection of a natural number $v$ inside GI $\Delta_{N}$ have a different probability $P\left(q_{s} \mid v\right)$ from $1 / q_{s}$ whatever the interval $A_{m-1} A_{m}$ in which happened to be the selection of $v$. But thus we observe that in this way a ran- 
dom interval $A_{m-1} A_{m}$ was chosen independently to the method of subdivision of GI $\Delta_{N}$. Therefore, because the method is arbitrary, we can select as method of subdivision the specific method of subdivision into $\delta_{0}, \delta_{1}, \delta_{3}, \cdots, \delta_{K(N)}$. This analysis easily leads to the conclusion that inversely now, selecting a random $\delta_{M}$, the probability $P\left(q_{s} \mid v\right)=1 / q_{s}$ will be stable inside this $\delta_{M}=R\left(\Delta_{N}\right)$. Proposition: (58).

We can go deeper, examining the minimum distances of any multiple mul. $q_{s}$ from the limits of $\delta_{M}=R\left(\Delta_{N}\right)$. That is to prove the distances between the boarders of a band $b_{s}(M)$ and the limits of $\delta_{M}$ are as well random. We will use the (42) in general form with (43) and with the proposition (46). For this let be one prime number defining the first limit of a random RSI:

$$
q_{M}=R\left[\left\{q_{M(i)}: i=1,2, \cdots, K\right\}\right]=R\left(N_{q}\right)
$$

and let be a prime number $q_{s}=R\left(Y_{M}\right)$. Thus initially we selected $q_{M}$ random in $N_{q}$ and not in N. Next, choosing the natural numbers $n_{s i}=R(N)$ let define other natural numbers by the relation:

$$
V_{s i}=n_{s i} q_{s}-q_{M(i)} \times q_{M(i)}
$$

Let be initially suppose that $V_{s i}$ is not random in $N$, that is $V_{s i}=n o t \cdot R(N)$. This is the hypothesis. Let be this as Proposition: (60)

Next, we can define random numbers in $\mathrm{N}$ as product each time of two random numbers, a) $q_{M(i)}$ and b) $N_{j}=R(N)$ :

$$
N_{i}=q_{M(i)}\left(q_{M(i)}\right)^{m(i)-1} \prod_{i \neq j=1}^{j=N_{0}}\left[q_{M(j)}\right]^{\mu(j)}=q_{M(i)} \times N_{j}, \quad\left\{q_{s}, \text { not } \mid N_{i}\right\}
$$

where $\left(q_{M(i)}\right)^{m(i)}$ is random in set $\left(N_{q}\right)^{m(i)}, N_{0}=R(N)$ : Multiplying the members of the relations (59) with $N_{j}$ of (61) we get:

$$
N_{j} V_{s i}=N_{s i} q_{s}-N_{i} \times q_{M(i)}
$$

But because $n_{s i}$ is random in (59) from the definition itself, we conclude that and $N_{s i}=N_{j} n_{s i}$ will be as well random in $N$ as product of two random selected natural numbers. Let be this as Proposition: (63)

We can write the general form of (42) for a random multiple $v$ of $q_{s}$, that is:

$$
P\left(v=m u l . q_{s} \| v=\text { mul. } q_{M(i)}\right)=P\left(v=m u l . q_{s}\right)=1 / q_{s}
$$

Here we use the random selections according to the definition (31) to study events in set $\mathrm{N}$ with the classical theory of probability. Next, we define that one relation $n=n_{1} q_{s}-n_{2} q_{M(i)}$ represents the (64) when, and only when, two of the three natural numbers $n, n_{1}, n_{2}$ are random in $\mathrm{N}$, and therefore on the basis of the probability theory from the independent prime's multiples (events) of the relation (64) we have as conclusion that and the third natural number of them as well will be random in N. Proposition: (65)

Combining the propositions (62), (63) and the fact that $N_{i}$ is also random in 
$\mathrm{N}$ (as we defined) we observe that accordingly to (65) the (62) represents the (64) and from this [taking in to account the (46)] we conclude that the first member $n=v=N_{j} V_{s i}$ will be random in N. But thus we conclude a proposition which contradicts to our hypothesis (60), because according to (60) the $V_{s i}$ is not random in $\mathrm{N}$ and thus the product of not random $V_{s i}$ with the random $N_{j}$ is not random in N. Conclusively we must reject the (60) and thus accordingly to (65) now the relation (59) represents as well the (64) which implies for the third number of (59) that $n_{2}=q_{M}=q_{M(i)}=R(N)$. We observe that selecting initially one $q_{M}=R\left(N_{q}\right)$ then we proved that $q_{M}=R(N)$ and therefore according to the relation (46) and to the familiar relation (43) of the number theory, for the minimum distance [remnants of divisions:

$\left.\left(V_{s i}\right)_{\min }=\Delta x_{\min }=v_{s}=n_{\min } q_{s}-q_{M}^{2}\right]$ in the random RSI in their set $D$ we will have the effect (of the events randomness) of the probability theory for independent events (multiples of primes) in $N$ :

$$
\begin{gathered}
\left\{V_{s i}=R(N), q_{s} \neq q_{M}\right\} \Rightarrow \\
v_{s}=\left[n_{s} q_{s}-q_{M}^{2}\right]_{\text {min }}=n_{\text {min }} q_{s}-q_{M}^{2}=R\left[\left\{1,2, \cdots, q_{s}-1\right\}\right]=R\left(I_{s}\right)
\end{gathered}
$$

In other words we result to the fact that because we proved that $q_{M}^{2}$ has been essentially selected random in the set $\mathrm{N}$ (as a product of two similar numbers random in $\mathrm{N}$ ) and not only in $N_{q} \times N_{q}$ then the minimum distance between this random place $\mu=q_{M}^{2}$ and the multiples of $q_{s}=R\left(Y_{M}\right)=R(N)$, and also $q_{s}=R(N)$ [because $\mu, M=R(N)$ ], will be obviously random in $I_{s}$, that is $\Delta x_{\min }=R\left(I_{s}\right)$. The same obviously is valid about $q_{M+1}^{2}$ of the other limit. So we proved that the distances between the limits of a band $b_{s}(M)$ and the limits of a random $\delta_{M}$, in which belongs this band, are random in $\mathrm{N}$. Proposition: (66).

Taking in to account the proposition (66) the previous proposition (58)has been absolutely patented, that is:

$$
P\left(q_{s} \mid v\right)=1 / q_{s}=\text { const (constant) in } \delta_{M}=R\left(\Delta_{N}\right)=R(N)
$$

This result is very important for the distribution of prime numbers in $\mathrm{N}$ (that concerns and $\mathrm{RH}$ ). That is, according to (67) the production of twins in random RSI obeys to the general statistics which is exclusively and only under to the control of the relations and inequalities proven in next Sections 4, 5 and nothing more.

The (67) implies that the definitions not gave catholic information for the place of $n_{\min } q_{s}$ relative to the multiples of other primes of the $Y_{M}$ that is without catholic information (CI) for changing the $P\left[q_{s} \mid \mu\right]$ of $\mu=R(N)$ when we introduced the new catholic information: $\mu=R\left[\delta_{M}=R(D)\right]$. And evidently the same will be by the refusal propositions, that is with $q_{s 1}, q_{s 2}=R\left(Y_{M}\right), \mu, v=R\left[\delta_{M}=R(D)\right]$ (where obviously the $Y_{M}, \delta_{M}$ are corresponding) we get the corresponding relation of the (41):

$$
\left(q_{s 1}, \text { not } \mid v\right)<>\left(q_{s 2}, \text { not } \mid \mu\right)
$$


The other case (39) $\mu \in \delta_{M+1}$ can't change the result, because according to the proposition (2) this limited twin is counting as well, and on the other hand and if it still was not so, evidently would be multiplied the multitude of real state twins by a factor which would be very near to $1,(0<f<1)$, and thus the result is again infinite, because the product of infinite with such $f$ is again infinite.

Finally, we point out that graphs: $M \leftrightarrow W(M)$ of the next function of phases into RSIs may present interest to the distribution of prime numbers:

$$
W(M)=\sum_{v=q_{M}^{2}}^{v=q_{M+1}^{2}-1}\left(\sum_{s=1}^{s=M} \cos \left[2 \pi v\left(1 / q_{s}\right)\right]\right)
$$

According to the propositions (31), (32), (46) of catholic (general) information the proof has been based exclusively on distribution which comes from general definition of twins that correspond to random selection which is the only representative of the statistical distribution of twins in the infinite multitude of the natural numbers.

\section{The New Proof of Relation (4.17) of First Article A and Optimizations}

We will omit the passage from relation (4.14) to relation (4.16) of the first article A and bridge it here with the proof that relation (4.17) [resulting from the general form (4.16) by $g=2$ ] is valid. Specifically, we will prove that factor $g$ in (24) of previous Section 2, which is its analytical form (4.16) in the first article A, makes valid the inequality (4.17) if we replace its value $g=2$ in relation (4.17) with a value between value $2-\sqrt{2} / 2<1.293 \cong 1.3$ and value 2 . That is we refer at paragraph 3 of section "The steps" at the beginning of this second article B. We will here use Figure 1 of Section 4 of first article A (as well in Introduction here again), together with relation (4.5), which is the same as relation (3.2) of Section 3, where this has been proved. Figure 1 shows two bands $b_{s}(M)=[\gamma, \zeta]$, $b_{\rho}(M)=[\alpha, \eta]$, where the first band is included in the second, with their limits $\gamma, \zeta$ and $\alpha, \eta$ respectively. There is also an eraser of $b_{\rho}(M)$ in position $\beta$ and another of $b_{s}(M)$ in position $\delta$, as well as the spectral zones $\gamma \delta, \delta \varepsilon, \varepsilon \zeta$ [with length $q_{s}$ for every one zone] of band $b_{s}(M)$. The relation (4.5) shows that the minimum number of these spectral zones, in which each band is subdivided, is at least 4 . However, in Figure 1 we presented only 3 spectral zones for reasons of economy of shape, as we explained in Section 4 of the article A. Each band is subdivided into spectral zones by its erasers, i.e. the multiples of the specific prime number to which this band corresponds. These are all the multiples (of its corresponding prime number) which are enclosed within the specific real silver interval (that is RSI) $\delta_{M}$ with limits $G, L$ where this band is formed each time, as we analyzed in the introduction earlier.

As we explained in the introduction earlier, there are only two ways of overlapping the bands. One is the way shown in Figure 1 (it is that of paragraph 4 in article $\mathrm{A}$ and in Introduction above here) in which one band is contained whole inside another band and we called this way of "not intersection way". While the 
other way is the way where the two bands are intersected between them and we called it the "intersection way".

We will not give a new Figure for this case, because this can be made immediately, where we will consider the band $b_{s}(M)=[\gamma, \zeta]$ not to be contained inside band $b_{\rho}(M)=[\alpha, \eta]$ but to be intersected by this. This can be done very simply by moving the boundary $\gamma$ of band $b_{s}(M)$ and placing it to the left of the boundary $\alpha$ of the other band $b_{\rho}(M)$. Thus in this case of the "intersection way" we will observe that the limits of two above bands have the order $G, \gamma, \alpha, \zeta, \eta, L$, while in previous case of "non intersection way" of Figure 1 the limits of these bands have the order $G, \alpha, \gamma, \zeta, \eta, L$. Where $G, L$ they are the limits (boundaries) of this silver interval. We will call this Proposition: (70)

We will now move to the beginning of Section 2 where we presented the sentence (22), where we defined the arithmetic length of an interval $l([m, n])=n-m+1$. We will also note that the relation (24) in Section 2 gives us the exact number of primes in interval $l([m, n]) \in \delta_{M}$ if this length is a multiple of the product of all prime numbers of the subsequence $Y_{M}$. E.g. in $\delta_{2}=[9,25)$ with subsequence $Y_{2}=\{2,3\}$ for its interval [13, 24] with arithmetical length $l([13,24])=24-13+1=12=(2 \times 3) \times 2$ we have $N_{[13,24]}=l([13,24]) \cdot P_{2}(1)=12(1-1 / 2)(1-1 / 3)=12 \times 1 / 3=4$ prime numbers. Really they are the prime numbers $13,17,19,23$ which are all the prime numbers of interval $[13,24]$. But the same does not happen if we select the $\delta_{2}=[9,25)$ where we calculate:

$$
N_{[9,24]}=l([9,24]) \cdot P_{2}(1)=(24-9+1)(1-1 / 2)(1-1 / 3)=16 \times 1 / 3 \cong 5.33
$$

The multitude of prime numbers inside $\delta_{2}$ is $5<16 / 3$. The reason is that the arithmetical length 16 now is not a multiple of product $(2 \times 3)=6$.

We call this last proposition of "the calculation of exact multitude of prime numbers", as Proposition: (71)

We will now define as a percentage arithmetical length for every silver interval a constant length $c$ equal to 1 , that is $c=1$ for every RSI and so and for its every corresponding $\delta_{M}^{\text {vir }}(g)$. We are talking in principle about $\delta_{M}^{\text {vir }}(g)$ where works exclusively the relation (24), as we said. Next let's then study at the relation (29) on the basis of relation (22) mentioned just before. Let us first set $g=1$ and let us assume that the RSI $\delta_{X}$ contains only two bands $b_{i}(X)$, $b_{j}(X)$. Such VSI $g=1$ is the $\delta_{2}^{\text {vir }}(1)$. Obviously only these same bands will be contained in every $\delta_{X}^{\text {vir }}(g)$. So this relation (28) takes the form (29) that is $\rho=(x+y)-x y$ for the $\delta_{x}^{\text {vir }}(1)$. If we zero the $x, y$ the $\rho$ will be zero and its entire percentage length $c=1$ will contain a fraction $\kappa_{0}$ of the prime numbers that will be due only to bands $b_{i}(X), b_{j}(X)$, and will be given by (24) with $g=1$. So we have: $\kappa_{0}=c P=c(1-0)(1-0)=1-0=1-\rho_{0}=1$.

This result simply means that by abolishing the two moving erasers $q_{i}$ -multiple, $q_{j}$-multiple [that correspond to the two bands $\left.b_{i}(X), b_{j}(X)\right]$ the whole arithmetical percentage length $c$ of $\delta_{M}^{v i r}(1)$ consists only of prime num- 
bers ( $\kappa_{0}=1$ of $c=1 \rightarrow 100 \%$ ). Let's now we enter only the $\rho_{1}=x+y$. We get $\kappa_{1}=1-(x+y)$. So now the fraction $\kappa_{0}$ has been changed to a new fraction $\kappa_{1}<1$ again of $c=1 \rightarrow 100 \%$, and so now will be $\kappa_{1} \rightarrow$ less than $100 \%$ of multitude of natural numbers of default $\delta_{X}^{\text {vir }}(1)$ or also of the same multitude of natural numbers of RSI $\delta_{X}$. This means that all deletions from the two erasers were made inside $\delta_{X}^{\text {vir }}(1)$ without them coinciding between them on some natural numbers of the $\delta_{X}^{\text {vir }}(1)$. However, this is not correct. What is important at this point is that $x+y$, and therefore $R_{M}(g)$ in the general case of (28), represents the fraction of $c=1$ of all deletions without degeneration, that is, without coinciding two deletions of different kinds of erasers between them somewhere, even only once. To get the correct virtual fraction from the above $\rho_{1}$ we have to subtract from $\rho_{1}=(x+y)$ the fraction of all the degenerate deletions that $K_{M}(g)$ represents inside the (28) and here, for our simplest case, this corresponds to the term $g^{2} x y=1^{2} x y=x y$ of (29). Therefore, finally we must write $\kappa=1-\rho=1-[(x+y)-x y]$. And for the multitude of prime numbers in $\delta_{X}^{\text {vir }}(1)$ we have: $N_{X}=d_{X} P_{X}(1)=\left(q_{X+1}^{2}-q_{X}^{2}\right) \kappa$. And more generally we must write the relation (28) for the "virtual clear density of deletion" $\rho$ for some $g \geq 1$ and for all bands of $\delta_{M}^{\text {vir }}(g)$. We understand that $R_{M}(g)$ of relation (28) represents the fraction $\rho_{M 1}$ of the deletions from all moving erasers of $\delta_{M}^{\text {vir }}(g)$ without coincidences between them, i.e. without degenerations. While $K_{M}(g)$ represents the fraction of all coincidences, i.e. the fraction of the number of degenerate cases [inside the $\delta_{M}^{v i r}(g)$ ] which must subtract of $\rho_{M 1}$. Thus we can see that subtract of "virtual degenerate deletion density" from the "virtual total deletion density" in (28) gives the clear fraction of deletions or the "virtual clear density of deletion" in the total space of $\delta_{M}^{\text {vir }}(g)$. Now we'll find the corresponding for a part (fraction) of space of $\delta_{M}^{v i r}(g)$. It is now easy to see that for an arithmetical length $l([m, n])$ of (22) within the total arithmetical length of $d_{M}$ of random silver interval $\delta_{M}$ the corresponding percentage length will be $l_{p}=l([m, n]) / d_{M}$ of $c=1$. So within this fraction $l_{p}$ of $c$ the fractions of $R_{M}(g), K_{M}(g)$ will be changed analogically to the smaller ones:

$$
r_{l M}(g)=l_{p} R_{M}(g), k_{I M}=l_{p} K_{M}(g)
$$

And obviously they will have the same meaning for $l_{p} \leq 1$ which they had the fractions $R_{M}(g), K_{M}(g)$ for the $c=1$ previously. We conclude that the fraction of the prime numbers in $l_{p}$ will be $l_{p} P_{M}(g)$ while the fractions of the deletions of the two types $R, K$ have been changed now to those is given from relation (72). We will now consider how (70) applies in the two relation cases (72) that refer to the interaction of only two bands:

$$
b_{s}(M)=[\gamma, \zeta], b_{\rho}(M)=[\alpha, \eta] .
$$

We will also use the relation (3.2) or (4.5) of article A, which is also presented in the 10 of "Nomenclatures" (section in Appendix at the end of this article B). In the first case of "not intersection way" of (70), at the beginning of this section 4 , we observe that the two bands $b_{s}(M), b_{\rho}(M)$ of RSI have overlap only 
within the segment $[\gamma, \zeta]$ and therefore only in this segment the relation (25) will be valid. The (24), (25) are valid for the entire space of the corresponding any VSI as we said, because in VSI these two bands have ideal overlap along the entire $G L$ length of silver interval. Therefore $q_{i}$-multiple, $q_{j}$-multiple moving erasers will not coincide anywhere in the two segments $\theta_{1}=[\alpha, \gamma]$ and $\theta_{2}=[\zeta, \eta]$ of RSI $\delta_{M}$. Therefore, in order to have the same clear deletions between RSI and VSI within these exactly sections $\theta_{1}, \theta_{2}$ of the "disorder" of RSI, the $K_{M}(g)$ inside these corresponding sections $\theta_{1}, \theta_{2}$ of VSI $g=1$ should be zero ("as it is potentially happening in its corresponding RSI") instead of the value calculated by the relation (29) that expresses the interaction between both bands. That is, based on (29) for (25) we should have for overlap in $\theta_{1}, \theta_{2}$ (of VSI 1) that $l_{p} K_{M}(1)=K_{M}(1)\left[l\left(\theta_{1}\right)+l\left(\theta_{2}\right)\right] / d_{M}=0$ and not $l_{p} K_{M}(1)=l_{p} x y>0$ that corresponds to calculations of (25), where the (29) hide inside its expansion, so that in the end to we have the same number of degenerate deletions in RSI $\delta_{M}$ and VSI $g=1$. Because in reality something this do not happens and we do only a hypothesis to find the way toward the asking VSI $g>1$. This is why on accordance (29) the "virtual clear density of deletions" is less than the corresponding "real clear density of deletions". According to what we said about the search for proper $g>1$ we should select the factor $g$ so that the new deletion density at new unknown VSI $g>1$ [instead of VSI $g=1$ ] $\rho=U(g)=g(x+y)-g^{2} x y$ increases over the deletion density $1(x+y)-1^{2} x y$ of VSI $g=1$ of (29) by an amount greater than $l_{p} x y$, which has been refer just before. So must be valid:

$$
\left[g(x+y)-g^{2} x y\right]-\left[1(x+y)-1^{2} x y\right]>l_{p} x y
$$

However, we can easily understand that for the percentage length $c=1$ the $l_{p}=\left[l\left(\theta_{1}\right)+l\left(\theta_{1}\right)\right] / d_{M}$ based on the (4.5) of the article A will satisfy the relation $l_{p}<1 / 4$, because based on this useful inequality (4.5), and symbolizing distances as $\alpha \gamma, \zeta \eta, G \gamma)$ etc., it will be:

$$
\theta_{1}+\theta_{2}=\alpha \gamma+\zeta \eta<G \gamma+\zeta L<q_{s}<G L / 4 \rightarrow c / 4=1 / 4
$$

Because in percentage lengths we have the contraction $G L \rightarrow c=1$. So finally, based on the above, and mainly to (73), (74), must be valid:

$$
\left[g(x+y)-g^{2} x y\right]-\left[1(x+y)-1^{2} x y\right]>x y / 4
$$

So that the relation (73) applies. If we repeat the same procedure for the other case of its "intersection way" of proposition (70), where the complete overlap of the two bands is now done in their common part $[\alpha, \zeta]$, following a completely corresponding procedure, we understand that the application (74) is now replaced by the requirement:

$$
\begin{aligned}
& \gamma \alpha+\zeta \eta<G \gamma+\gamma \alpha+\zeta \eta+\eta L=G \alpha+\zeta L<q_{\rho}+q_{s} \\
& <[G L / 4]+[G L / 4]=G L / 2 \rightarrow c / 2=1 / 2
\end{aligned}
$$

Because again, based on (4.5) of article A, we will have $G \alpha<q_{\rho}<G L / 4$ and $\zeta L<q_{s}<G L / 4$ for $b_{\rho}(M), b_{s}(M)$ respectively. So (75) leads, as before, to 
the requirement that it is sufficient for the relation:

$$
\left[g(x+y)-g^{2} x y\right]-\left[1(x+y)-1^{2} x y\right]>x y / 2
$$

So that the relation (73) applies again. Relations (75), (77) both have the sufficient condition the (77) for the validity of (73) which we demand for proper VSI $g>1$ [or $\delta_{M}^{v i r}(g)$ ]. In this way we showed that (77) is the sufficient condition for the asking VSI $g>1$ so that it has a greater "virtual clear density of deletion" than the "real clear density of deletion" of RSI $\delta_{M}$ and therefore the relation (24) to give for this proper VSI $g>1$ less multitude of prime numbers than the corresponding random RSI $\delta_{M}$ for all $M$. i.e. to be valid the (77) for the required VSI $g>1$ for all $M$. Because the probability function in (24) is decreasing as $g$ increasing, and just that is we need, that is, a less multitude of prime numbers to proving equation (4.17) for proving next the (4.18) in first article A. But in fact in the first article A, we did just that. Below now we'll calculate a sufficient critical minimum value of proper VSI $2>g>1$ and as we promise we'll prove this is:

$$
1.3 \leq g_{\text {proper }}<2
$$

We only used two bands. However, since thus VSI $g>1$ increases its virtual clear density of deletion per two bands, it follows that this density will increase for all bands, because the interaction in the overlaps with a third band (and so on) does not cancel the result (77) of the two bands. This we will prove. In other words we will prove little below that the proper increment of "virtual clear density of deletion" in comparison to "real clear density of deletion" per any pair of bands produces the same proper comparative increment (of "virtual clear density of deletion" in comparison to "real clear density of deletion") from the general overlap of all bands together. Proposition: (79).

Let us prove the above (79) analytically: For every RSI $\delta_{M}$ inevitably will exist positive rational factors, that is $G_{1 M}, G_{2 M}, \cdots, G_{M M} \in Q^{+}$with the restriction $0<G_{i M}<q_{i}$ so we can have a correct calculation of exactly probability (or fraction) of prime numbers in RSI $\delta_{M}$, and $\forall M \in N$, in correspondence to (24):

$$
P_{M 0}=\prod_{j=1}^{M_{v}} F_{j}\left(G_{j M}\right)=\left(1-\frac{G_{1 M}}{2}\right)\left(1-\frac{G_{2 M}}{3}\right) \cdots\left(1-\frac{G_{M M}}{q_{M}}\right)
$$

The reason is that to every $b_{j}(M)$ band always corresponds some $D_{j M}$ value of a rational number that represents the rational number of deletions of moving eraser $q_{j}$-multiple as a result which took into account also its coincidences by the all other moving erasers $q_{i}$-multiples. And so will exist always one suitable $G_{j M}$ rational number that satisfies the equation $F_{j}\left(G_{j M}\right)=D_{j M}$. Based on the relation (67) according to (3) we will prove in Section 5 that the $P_{M 0}$ can be considered constant on each $v \in \delta_{M}$ during the counting of the elements of $\delta_{M}$. Next, generalizing the relation (2.B3) we get the probability for the corresponding default VSI $g=1$ :

$$
P_{M}(1)=\prod_{j=1}^{M_{V}} F_{j}(1)=\left(1-\frac{1}{2}\right)\left(1-\frac{1}{3}\right)\left(1-\frac{1}{5}\right) \cdots\left(1-\frac{1}{q_{M}}\right)
$$


Now the relation (24) gives the probability of the asking VSI $g>1$ :

$$
P_{M}(g)=\prod_{j=1}^{M_{v}} F_{j}(g)=\left(1-\frac{g}{2}\right)\left(1-\frac{g}{3}\right)\left(1-\frac{g}{5}\right) \cdots\left(1-\frac{g}{q_{M}}\right)
$$

Taking in to account the relation (82) we conclude that to be valid the (79) is sufficient be valid every inequality of the type:

$$
\left(1-\frac{G_{i M}}{q_{i}}\right)\left(1-\frac{G_{j M}}{q_{j}}\right)>\left(1-\frac{g}{q_{i}}\right)\left(1-\frac{g}{q_{j}}\right)
$$

The proof is that by correlating the relations (80), (82) on the basis of relation (15) [which we before proved in the part A of Introduction here] we conclude that the set of inequalities of (83) will implies the general inequality:

$$
P_{M 0}>P_{M}(g), g>1
$$

These all happens because we can't correlate one by one the factors of parenthesis between the relations (80) and (82), but according to the previous analysis two by two. Therefore thanks to (15) we prove the (79) as we promised. And from the other hand the (83) is valid when, and only when, the "real clear density of deletion" that represent the first member of (83) be done smaller than the "virtual clear density of deletion" of the second member and something such happens when the above condition (77) is valid, as we proved before using the default VSI $g=1$ as one mediator of the correlation all possible pairs from factors $F_{j}$ between the relations (80), (82).

To make this clear we can expand the members of (83) so to have the expression $1-\rho_{0 . i j}>1-\rho_{i j} \Leftrightarrow \rho_{0 . i j}<\rho_{i j}$ that is equivalent to (77).

From the above we conclude that the main condition of finding the asking proper $g$, [for proving of (78)] is the inequality (77) of $g$, that takes the form:

$$
\left((g-1) x-\frac{1}{2} g^{2} x y+\frac{x y}{4}\right)+\left((g-1) y-\frac{1}{2} g^{2} x y+\frac{x y}{4}\right)>0
$$

This relation (85) has as sufficient relations the next two:

$$
(g-1) x-\frac{1}{2} g^{2} x y+\frac{x y}{4}>0,(g-1) y-\frac{1}{2} g^{2} x y+\frac{x y}{4}>0
$$

We point out that because we don't need all proper values of $g$, but only some above the 1, we looking for only sufficient inequalities to be valid the (77) and not the sufficient and necessary inequalities for (77). The two inequalities of (86) are equivalent each other because the two factors $x=1 / q_{i}$ and $y=1 / q_{j}$, which have been defined just above the (29), are both less or equal to $1 / 2$, because are $q_{i}, q_{j} \geq 2$ as prime numbers:

$$
x=1 / q_{i} \leq 1 / 2 \text { and } y=1 / q_{j} \leq 1 / 2
$$

So is sufficient to solve one of them as to $g$ in (86), e.g. the second of them, so eliminating the $y$ we get:

$$
2 x g^{2}-4 g+(4-x)<0
$$

If the discriminant $\Delta_{x}$ of quadratic inequality (88), as function of $x$, is nega- 
tive the (88) is impossible. Therefore must be:

$$
\Delta_{x}=8\left(x^{2}-4 x+2\right)>0 \Leftrightarrow
$$

$\{x>2+\sqrt{2}$ that is rejected from (87) or $0<x<2-\sqrt{2} \cong 0.6$, that is accepted\}.

Putting $D=\sqrt{4-2 x(4-x)}$ we conclude that for (88) is sufficient to be:

$$
x^{-1}(1-D / 2)=f_{L}(x) \leq g \leq f_{R}(x)=x^{-1}(1+D / 2)
$$

The derivative is:

$$
f_{L}^{\prime}(x)=(2-2 x-D) /\left(x^{2} D\right)
$$

So to be $f_{L}(x)$ increasing function of $x$ must be $f_{L}^{\prime}(x)>0$ and so must be:

$$
-2 x+2>\sqrt{4-8 x+2 x^{2}}=D
$$

But we percept that $-2 x+2>1>0, \forall x \in(0,1 / 2]$, so for the validity of (92) as long as it is valid $(-2 x+2)^{2}>4-8 x+2 x^{2}$, which is true. We conclude that the (92) is valid and so the function $f_{L}(x)$ is increasing function of $x$. Similarly we can see that for (90) the function $f_{R}(x)$ is a decreasing function of $x$. Moreover symbolize the decreasing function as $\downarrow$ we have the properties: $\downarrow+\downarrow=\downarrow$, $\downarrow \times \downarrow=\downarrow$, and so for the two terms of $f_{R}(x)$ we will immediately have: $\downarrow+(\downarrow \times \downarrow)=\downarrow+\downarrow=\downarrow$, and therefore the $f_{R}(x)$ is decreasing. The combination of the two diagrams of the functions $f_{L}(x), f_{R}(x)$ of the above relation (90) gives a beautiful graph of their mixing just below in Figure 2.

The diagrams of functions $f_{L}(x), f_{R}(x)$ are meeting at the points:

$$
(2-\sqrt{2}, 1 /(2-\sqrt{2})),(2+\sqrt{2}, 1 /(2+\sqrt{2}))
$$

Because $f_{L}(x)$ is increasing and $f_{R}(x)$ is decreasing as the $x$ increasing we finally conclude that for the domain $(0,1 / 2]$ of the $x$ any value of $g$ that satisfies (4B .14) should be located within the area defined by the maximum value of $f_{L}(x)$ that is $f_{L, \max }(x)=f_{L}\left(x_{\max }\right)=f_{L}(1 / 2)$ and the minimum value of $f_{R}(x)$ that is $f_{R, \min }(x)=f_{R}\left(x_{\max }\right)=f_{R}(1 / 2)$.

Therefore:

$$
g \in\left[f_{L}(1 / 2), f_{R}(1 / 2)\right]=[2-(\sqrt{2} / 2), 2+(\sqrt{2} / 2)]
$$

From the other hand, like a check, the derivative of its first member $\varphi(x, y)$ of the relation (85) will be increasing with respect to $g$ if, and only if, is:

$$
\varphi^{\prime}(x, y)=\varphi_{g}=(x+y)-2 g x y>0 \text { or } g<(x+y) /(2 x y) \geq 1+1=2
$$

Because $x, y \leq 1 / 2$. So since it is always $(x+y) /(2 x y) \geq 2$, it is enough for the validity of the sufficient condition (85) [to be valid the (77) and so on] to choose one $g$ equal or less than 2 and in accordance to the (93). Combining this maximum 2 of $g$ [which we used in (4.17) of the article A] with the above minimum of $g$ from (93) we conclude that for sufficiency of our conditions we have to select the factor $g$ from the space:

$$
2-(\sqrt{2} / 2) \leq g \leq 2
$$




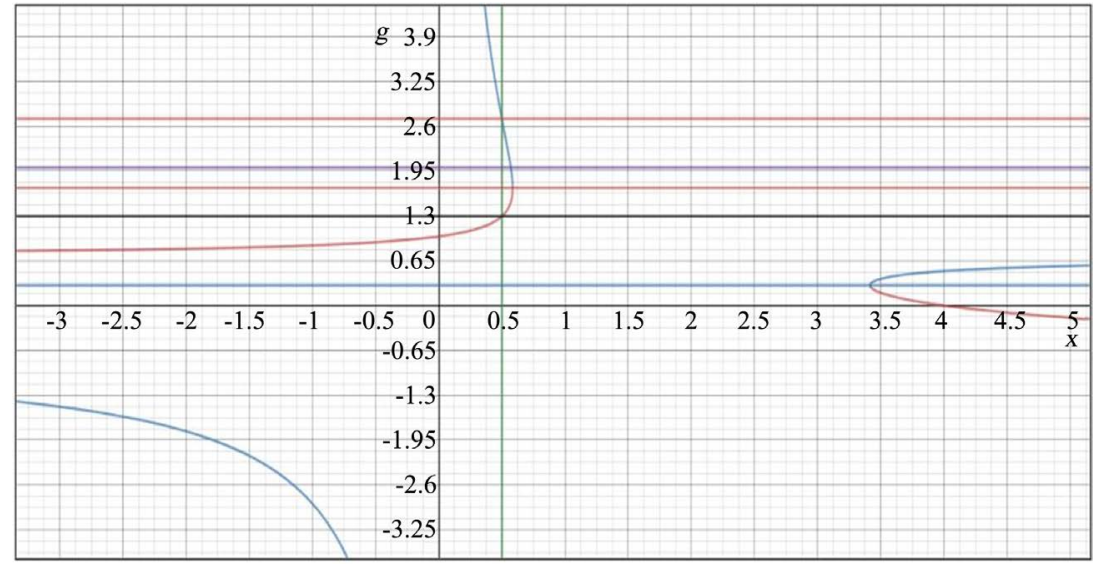

Figure 2. Diagrams of $f_{L}, f_{R}$ functions.

So the relation (85) or its equivalent (77) to be valid. Therefore the minimum value of $g$ will be: $g=2-(\sqrt{2} / 2)=1.293 \cdots<1.3$.

And so finally we prove that the relation (78) does apply. We understand that the choice of VSI $g=2$ case of first article A in the relation (4.17) drives to take place the reduction of multitude of prime numbers and the reduction of multitude of twins in all VSI $g=2$ relative to primes and twins multitudes inside the corresponding RSI $\delta_{M}$ intervals. And therefore the calculations and the proof after (4.17) in the article A be correct. But for the completeness of this proof we must analyze something more, and this we will make just bellow. The relation (24) corresponds to the random VSI $g=2$ and then because for $g=2 \in[2-(\sqrt{2} / 2), 2]$ the (84) for this VSI $g=2$ will conclude fewer prime numbers than the corresponding RSI $\delta_{M}$. Subsequently for this VSI $g=2$ putting this value of $g$ in (24) we get:

$$
P_{M}(2)=\prod_{j=1}^{M_{v}} F_{j}(g)=\left(1-\frac{2}{2}\right)\left(1-\frac{2}{3}\right)\left(1-\frac{2}{5}\right) \cdots\left(1-\frac{2}{q_{M}}\right)=0
$$

We percept that this probability is zero and therefore we can't use the value $g=2$ for the factor $F_{1}(g)=(1-g / 2)$. We must therefore substitute the value $g=2$ especially only for the first factor $F_{1}\left(g_{1}\right)$ with another value $g_{1}$ that we must calculate. However, this will result in a hybrid VSI $g_{1}, 2$ where the $b_{1}(M)$ band will have a different value of $g$ of its $q_{1}$-multiples eraser in relation to the erasers of all the other bands that will have the same value $g=2$. Here emergences a new mathematical phenomenon. Based on the relation (95) that we found, if we eliminate the first band $b_{1}(M)$, all the other bands in pairs will increase their "virtual clear density of deletion" in relation to their respective pairs in the RSI $\delta_{M}$. Therefore for this to happen with all bands without exception, each pair, that construct the band $b_{1}(M)$ by $g=g_{1}$ with any other band $b_{s}(M)$ by $g=g_{s}=2, s \neq 1$, must do the same thing, that is, increases its "virtual clear density of deletion" relative to its corresponding pair in the RSI $\delta_{M}$. Therefore we will go to relation (29) and we will ask for its corresponding relation the new form generally for $g_{i}, g_{j}$. So we will have now: 


$$
\begin{aligned}
& {\left[1-\left(g_{i} / q_{i}\right)\right]\left[1-\left(g_{j} / q_{j}\right)\right]} \\
& =\left(1-g_{i} x\right)\left(1-g_{j} y\right)=1-\left(g_{i} x+g_{j} y-g_{i} g_{j} x y\right)=1-\rho_{i j}
\end{aligned}
$$

The new general "virtual clear density of deletion" is $\rho_{i j}$ and refers to pairs $b_{i}(M), b_{j}(M)$, and will be:

$$
\rho_{i j}=g_{i} x+g_{j} y-g_{i} g_{j} x y
$$

The relation (97) now replaces the relation (29). Therefore, for the search of $g_{1}=g_{i}$, which concerns exclusively the interaction of the $b_{1}(M), b_{j}(M)$ bands of HVSI $g_{1}, 2$ relative to corresponding RSI (because the interaction between the all other pairs of bands with the same $g=2$ has been investigated just before) we will accept in below the general cases of "equal or greater", "equal or less" instead of previous "greater" and "less" correspondingly, because the probable equalities instead of the previous sufficient inequalities between the "virtual clear deletion densities" obviously do not change our conclusions and thus we will have successive:

The (97) instead of (29) and therefore we have the relation:

$$
\begin{array}{r}
\left(g_{i} x+g_{j} y-g_{i} g_{j} x y\right)-(x+y-x y) \geq x y / 2 \text { instead of }(77) \text { and } \\
\left(g_{i}-1\right) x-\left(g_{i} g_{j} x y / 2\right)+(x y / 4) \geq 0,\left(g_{j}-1\right) y-\left(g_{i} g_{j} x y / 2\right)+(x y / 4) \geq 0
\end{array}
$$

Instead of (86) and so we have:

$$
2 g_{i} g_{j} y-4 g_{i}+(4-y) \leq 0, \quad 2 g_{i} g_{j} x-4 g_{j}+(4-x) \leq 0
$$

Instead of (88). And accordingly to the above we must put $g_{i}=g_{1}, g_{j}=2$ into (99) and thus the relations (99) result:

$$
g_{1} \leq(y-4) /[4(y-1)] \text { and } g_{1} \leq 0.25+(1 / x), x, y \in(0,1 / 2]
$$

For the first relation of (100) we have:

$G(y)=[(y-4) /[4(y-1)]]^{\prime}=3 /\left[4(y-1)^{2}\right]>0$ and so this is increasing function, that is $G(y) \uparrow$, and next using the first relation of (100) we find the result:

$$
g_{1} \leq G_{\min }(y)=G\left(y_{\min }\right)=G(0)=1
$$

The 2st of $(100)$ gives: $H^{\prime}(x)=(0.25+1 / x)^{\prime}=-1 / x^{2}<0 \Rightarrow H(x) \downarrow$

And using the last relation with the second relation of (100) we find the result:

$$
g_{1} \leq H_{\min }(y)=H\left(y_{\max }\right)=H(1 / 2)=2.25
$$

The (102) is compromiser because for the value $g_{1}$ is known that $g_{1}<2$ and consequently the (102) don't give us extra information for the asking value $g_{1}$. Combining (101), (102) we conclude that one allowed value for the asking sufficiency is:

$$
g_{1}=1
$$

We must point that the relations (94) and (103) gives us only some of useful 
values of $g$ but not all values, because we moved on a very open way for proper sufficient conditions (which imply these values $g_{1}=1, g=2$ ) and not on equivalent relations. Therefore the result (101) doesn't mean that values of $g$ greater than 1 re excluded. Correcting the (95) by use of (103) we have the total probability of the hybrid VSI which in below we'll symbolize as HVSI 1, 2:

HVSI 1, 2:

$$
P_{M}=\left(1-\frac{1}{2}\right)\left(1-\frac{2}{3}\right)\left(1-\frac{2}{5}\right) \cdots\left(1-\frac{2}{q_{M}}\right)>0
$$

On the basis of all the above we proved that the above HVSI 1, 2 has greater "virtual clear density of deletion" (from the interaction of all its bands together, because we examined all possible cases per two bands) in relation to the corresponding RSI $\delta_{M}$ and therefore we proved that this random (that is for the random value $M$ ) HVSI 1, 2 will have fewer prime numbers of its corresponding RSI $\delta_{M}$. Let be this the Proposition: (105).

In corresponding relation (4.17) of our first article A we used there as value $g_{1}=1.75>1$ instead of the value of above relation (103), but something such do not change the final result because $0.5 \times \infty=0.125 \times \infty$, and for this below in Section 5 we will repeat the part of calculations after the relation (4.17) until the relation (4.22) of the first article A but now with (104), not only for this trivial reason, but mainly to give to the reader a complete image of our analysis here. The result and the form of calculations are same.

\section{Organization of the Steps and the Final form of the Proof}

Let's gather the steps of the proof.

1) We have shown the proposition (105) that random RSI contains more prime numbers than the corresponding ideal HVSI 1, 2. The probabilities of the prime number occurrence in any random position $v$ of a natural number inside these RSI $\delta_{M}$ and HVSI 1, 2 will be given by the (80) and (104) respectively.

2) We have said that the stability of $P_{M 0} \quad \forall v \in R\left(\delta_{M}\right)$ of the relation (80) drives on basis of (3) to the stability of the corresponding probability of $P_{M 0, t w i n}=P_{M 0} P_{M 0}^{\prime}$ of the twins within the same $\delta_{M}$. This stability of $P_{M 0}$ can be proved as follows. Let be $\mu, v \in R\left[\delta_{M}=R(D)\right]$. If $q_{s} \in Y_{M}=\left\{2,3, \cdots, q_{M}\right\}$ then due to the random choices we will have $P\left(q_{s} \mid \mu\right)=P\left(q_{s} \mid v\right)=1 / q_{s}$ according to (67). But on the other hand, on the basis of $\left(q_{s 1} \mid v\right)<>\left(q_{s 2} \mid \mu\right)$ we conclude that the cancellation of a measured deletion $q_{s} \mid \mu$ from the $q_{s}$ -multiples because it has an overlap of another eraser $q_{k}$-multiples of the deleting $q_{k} \mid \mu$, will have the same probability as the cancellation of a measured deletion $q_{s} \mid v$ from the $q_{s}$-multiples because it has an overlap (coincidence) of another eraser $q_{k}$-multiples of the deletion $q_{k} \mid v$. We observe that both the deletions of a multiple of type $\mathrm{P}$ and its cancellations of type $\mathrm{K}$ of the relation (28) are drives to the equal probabilities for random positions of $\mu, v \in R\left(\delta_{M}\right)$. And according to (68) we have and the symmetrical results for the not divisibili- 
ty.

Therefore, the two catholic events $\mathrm{P}, \mathrm{K}$, that determine the probability that the $\mu, v$ are prime numbers imply on the basis of (31), (32) that the $\mu, v$ would have equal probabilities to be prime numbers into the same $R\left(\delta_{M}\right)$ :

$$
P\left(v=\text { prime } \in \delta_{M}\right)=P\left(\mu=\text { prime } \in \delta_{M}\right)=k=\text { const }
$$

That is, the (106) catholically (generally) obeys exclusively and only to the relation (81) and to the catholic (general) inequalities which we proved for (80). In other words the factors $G_{i M}$ of the relation (80) in random $\delta_{M}\left[\delta_{M}=R(D)\right]$ will have random values obeying only to the general restrictions of mathematical necessities as it is $0<G_{i M}<q_{i}$, and inequalities we proved etc. The constant number $k$ of the above relation (106) as well the corresponding as well constants $k_{g}, k_{H}$ are given by (80), (82), (104) depending on what kind of silver interval we are referring to each time. By the definition of the random RSI the previous proposition (106) essentially comes from the missing of catholic information for special relations between places of bands inside this $R\left(\delta_{M}\right)$. In other words we have shown that the probability of a prime can be considered constant within a randomly selected $\delta_{M}$, as well in its corresponding ideal HVSI 1,2 from its definition because the HVSI 1, 2 is ideal. And according to the (106) we conclude that the every twin would have constant probability in the random RSI as well in ideal HVSI 1, 2 as we suppose in the calculations of the relation (5) in the introduction. Because the constancy of probability of prime numbers is the only proposition which influence absolutely on the basic relation (5) method of calculations below according to the classical theory of probabilities and statistics and nothing more.

3) We have defined the "random selection" in (31) of the Section 3. In the below we use the basic definitions in 1 and 5 etc of "Nomenclatures" (section in Appendix at the end) and the part A of Introduction at the beginning of this article B:

$$
P_{M 0}, P_{M}, P_{M 0}^{\prime}=\xi_{M 0} P_{M 0}, P_{M}^{\prime}=\xi_{M} P_{M}, \quad n=N_{M 0}, \quad N_{M 0, t w i n}, N_{M}, N_{M, t w i n}
$$

We conclude on the basis of (3) that in a random RSI the probability of a twin will be:

$$
P_{M 0, t w i n}=P_{M 0} P_{M 0}^{\prime}=\xi_{M 0}\left(P_{M 0}\right)^{2}=\left(P_{M 0} / P_{M}\right)^{2} \xi_{M 0}\left[P_{M}\right]^{2}, \forall M \in N
$$

But the proposition (105) gives

$$
P_{M 0} / P_{M}>1
$$

Now first we observe that if $n=N_{M 0} \geq 2$ then the relations (13), (107), (108) imply:

$$
P_{M 0, t w i n} \geq(1 / 2)\left[P_{M}\right]^{2}, \quad \forall M \in N \| n \geq 2
$$

But every special case with $n=N_{M 0} \leq 1$ results to $P_{M 0, t w i n}=0$ and its corresponding $P_{M}>0$ and so for these cases the (109) don't be valid. Therefore we must exclude these cases $n=N_{M 0} \leq 1$ from the applications of the relation (109) to the relations (123), (127) below. 
We must examine the other cases $n=N_{M 0} \leq 1$. For this we introduce the symbolisms:

$$
\begin{gathered}
N_{0}(M)=N_{M 0}, \quad f_{M}=N_{0}(M) / N_{0}(M-1), \quad \mathrm{K}_{M}=q_{M}^{2}-q_{M-1}^{2}, \\
\Lambda_{M}=q_{M} / q_{M-1}, \quad \mathrm{~A}_{M}=\mathrm{K}_{M+1} / \mathrm{K}_{M}, \quad \mathrm{~B}_{M}=\left[1-\left(1 / q_{M}\right)\right]=1 / \lambda_{M}
\end{gathered}
$$

We also define that "A proposition Pr will be statistically valid (SV) in a set $A$ with frequency $\omega_{v}$, symbolizing this as $\operatorname{Pr}\left(\omega_{v} \backslash \backslash A\right)$, when, and only when the $\operatorname{Pr}$ is valid on a fraction greaten than $\omega_{v}$ into the elements of the random selected subsets $A_{i}$ of $A$, which evidently for every $A_{i}$ are produced by random selections of elements from $A$ with a corresponding random (changeable every time) multitude $m_{i}$ under the condition that the random natural number $m_{i}$ to meet the special rules for the control of the truth of $\operatorname{Pr}$. If the $A$ has infinite number of elements then the statistics must provide the level of importance $\varepsilon=1$ (that is $100 \%$ certainty and not $\varepsilon=0.01$ etc) for the safety of the result

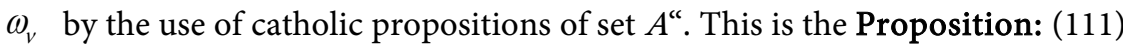

If is valid $\operatorname{Pr}(0.5 \backslash \backslash A)$ we will agree below to saying that the proposition $\operatorname{Pr}$ is simply statistically valid in set $A$. This is the Proposition: (112).

If the fraction $f_{M}$ is greater than 1 with $M=R(N)$, then the number of $n=N_{0}(M)$ of primes in RSIs $\delta_{M}$ will be a statistically ascending sequence of $M$ and because we know the initial values of $N_{0}(M)$ for finite values of $M$ we can percept that the majority of RSIs $\delta_{M}$ in their set $D$ must have $n=N_{0}(M)>2$. We will prove it by proving first that $f_{M}>1$. According to the above definitions (110) and 1, 5 of Nomenclatures (section in Appendix at the end of this article B) etc., we have: $N_{0}(M)=P_{M 0} d_{M}$ and so we must examine the validity of the relation

$$
f_{M}=\mathrm{A}_{M} \mathrm{~B}_{M}>1
$$

So for be valid the (113), is enough to be valid

$$
\mathrm{K}_{M+1}>\lambda_{M} \mathrm{~K}_{M} \text { or } \Lambda_{M+1}^{2}+\lambda_{M} \Lambda_{M}^{-2}>\lambda_{M}+1
$$

Initially comparing the numerator with denominator in $\mathrm{A}_{M}$ on the (113) and having in mind the logarithmic theorem of prime dilution, as more as the differences between successive primes increasing, we have the intuition that the above is valid, because from the other hand the factor $B_{M}$ must take values about 1 in contradiction to $A_{M}$ that must take values very greater than 1 . Let us examine it. From the proven theorem of prime numbers we know that the expected number of primes in interval $\{\Delta v\}=\left(q_{M+1}, q_{M}\right]$ by continuous and discrete values of the natural number $v$ and measure of interval $\Delta v=\Delta q_{M}=q_{M+1}-q_{M}$ will be:

$$
\Delta q_{M} / \ln \left(q_{M+1}\right)<I_{M}=\sum_{v=q_{M}}^{v=q_{M+1}}[1 / \ln (v)]<\Delta q_{M} / \ln \left(q_{M}\right)
$$

The above sum in (115) is referred often in form of an integral. Next for the above relation (115) we put: 


$$
u_{M}=\left(\ln q_{M}\right) / q_{M}, k_{M}=\left(\Delta q_{M} / \ln q_{M}\right)-I_{M}>0
$$

Because inside to the $\{\Delta x\}=\left(q_{M}, q_{M+1}\right]$ exists only one prime number (the $\left.q_{M+1}\right)$ we conclude that we can put $I_{M}=1$ and so the (116) gives:

$$
\Delta q_{M} / q_{M}=u_{M}\left(k_{M}+I_{M}\right)=u_{M}\left(k_{M}+1\right) \text { or }\left(q_{M+1}-q_{M}\right) / q_{M}=u_{M} k_{M}+u_{M}
$$

and then $\Lambda_{M+1}=1+u_{M}+u_{M} k_{M}$. Next for the other term $\Lambda_{M}^{-1}$ of (114) we observe an inverse inequality, that is $\Delta q_{M-1} / \ln q_{M}=\left(q_{M}-q_{M-1}\right) / \ln q_{M}<I_{M-1}$ and then putting:

$$
\left(q_{M}-q_{M-1}\right) / \ln q_{M}+k_{M}^{\prime}=I_{M-1}=1
$$

similarly we get the relation:

$$
\left(q_{M}-q_{M-1}\right) / q_{M}=-u_{M} k_{M}^{\prime}+u_{M}
$$

that results to $\Lambda_{M}^{-1}=1-u_{M}+u_{M} k_{M}^{\prime}$. Substituting $\Lambda_{M+1}, \Lambda_{M}^{-1}$ in (114) for the validity of the relation(114) is sufficient to be valid:

$$
F\left(k_{M}, k_{M}^{\prime}\right)=\left(1+u_{M}+u_{M} k_{M}\right)^{2}+\lambda_{M}\left(1-u_{M}+u_{M} k_{M}^{\prime}\right)^{2}>\lambda_{M}+1
$$

For the (117) first we will examine the:

$$
F(0,0)>\lambda_{M}+1
$$

Expanding the (117) is equivalent to $u_{M}>2\left(\lambda_{M}-1\right) /\left(\lambda_{M}+1\right)$ which is transformed to $q_{M}>\exp \left[q_{M} /\left(q_{M}-0.5\right)\right]<e^{2}$ which is valid $\forall M>4$. Next easily we observe that the function $F\left(k_{M}, k_{M}^{\prime}\right)$ in (117) is as well ascending of $k_{M}, k_{M}^{\prime}$ and so the relation (117) will be valid for all values of $k_{M}, k_{M}^{\prime}$ because these take positive values from their definitions. If we use the correct values $I_{M}, I_{M-1} \neq 1$, then similarly we result that for the validity of the relation (114) is sufficient to be valid:

$$
\left[\mathrm{d}\left(\Lambda_{M+1}^{2}\right) / \mathrm{d} I_{M}\right]>\left[\mathrm{d}\left(\Lambda_{M}^{-2}\right) / \mathrm{d} I_{M-1}\right],
$$

so the first member of the (117) to be ascending sequence of $M$. Or equivalently $\left[I_{M} q_{M+1}\right] /\left[I_{M-1} q_{M-1}\right]>1$. But this last result under examination is valid because its numerator statistically is greater than its denominator and the obvious reason is that the sequence $I_{M} q_{M}<I_{M} q_{M+1}$ is statistically ascending. Indeed, the $I_{M}$ counts the primes (with values here about 1 ) and thus is changing statistically very slowly than the corresponding increment of prime $q_{M}$ because the prime numbers dilute as their values increase more and more as they tend to the infinite. We conclude that the (114) is valid and therefore the (113) is as well valid. So we proved the relation (113).

Based on (111) let $\omega_{0}, \omega_{1}, \omega_{2}, \cdots \geq 0$ are the frequency's values with which $\delta_{M}$ of $D$ are displayed statistically and have respectively multitudes $n=N_{0}(M)=0,1,2,3, \cdots$ of their prime numbers. Let initially hypothesize that $0=\omega_{2}=\omega_{3}=\omega_{4}=\cdots$. Let be this as hypothesis Hyp-2. This could mean, for example, that within the infinite number of $\delta_{M}$ of the set $D$ only finite populations with $n=N_{0}(M) \geq 2$ appear. So based on Hyp-2 for the cases $n=N_{0}(M)=0,1$ inevitably will be $\omega_{0}+\omega_{1}=1$. According to the (113) that we 
proved will be valid for $\omega_{0}, \omega_{1}$ that $N_{0}(M+1)>N_{0}(M)$, and therefore statistically and with a level of security $\varepsilon=1$ (i.e. 100\%), we can claim that more than half of $\delta_{M}$ in $D$ will have $n=N_{0}(M) \geq 1$. That is, according to (112) this proposition $n=N_{0}(M) \geq 1$ will be statistically valid in $D$ if Hyp-2 is true in $D$. The reason is obviously that all $\delta_{M}$ with $n=N_{0}(M)=0$ have successors $\delta_{M+1}$ and therefore based on (113) we proved before, this will happen. That is, based on the definition (111) defining as $\operatorname{Pr}=\{n \geq 1\}$ the $\operatorname{Pr}(0.5 \backslash \backslash D)$ will apply. We therefore conclude that $\omega_{0}<\omega_{1}$, and after we said due to Hyp-2 it is valid $\omega_{0}+\omega_{1}=1$, we will have $\omega_{1}>1 / 2>\omega_{0}$. Similarly, all $\delta_{M}$ with $\omega_{1}>1 / 2$ and corresponding $n=N_{0}(M) \geq 1$ will have successors $\delta_{M+1}$ in $D$ for which the statistical relation (113) that is $N_{0}(M+1)>N_{0}(M)$ will be valid and with security $\varepsilon=1$ (i.e. $100 \%$ ) due to its universal power (113). So, similar to before, we have statistically populations of RSIs $\delta_{M}$ with $n=N_{0}(M) \geq 2, M=R(N)$ with occurrence frequencies $\omega_{2}>(1 / 2) \omega_{1}$ and again $\varepsilon=1$. And after we said $\omega_{1}>1 / 2$ it will finally be $\omega_{2}>(1 / 2)(1 / 2)=(1 / 2)^{2}$ and with $\varepsilon=1$ (i.e. $100 \%$ ) due to the catholic validity of the relation (113) in $D$. Therefore we proved that the hypothesis Hyp-2 is not valid and thus is rejected.

We conclude that there is a non-zero value $\omega_{2}$ for RSI $\delta_{M}$ which all have $n=N_{0}(M) \geq 2$. Proposition: (119).

We now have three useful conclusions:

1) Based on the relations (13), (107), (108), (119) we conclude that the inequality (109) will be valid in a non zero fraction $\omega^{*}>0$ of the total population of intervals $\delta_{M}$ and with random distribution of $M$ values concerning this population, due to the lack of catholic (general) information for some other distribution of $M$. Proposition: (120).

2) And finally using the (113) we have $\omega_{v}>\omega_{v-1} / 2, v=1,2, \cdots, v$ and multiplying these $v$ inequalities by members we result to $\omega_{v}>(1 / 2)^{v}$, $v=1,2,3, \cdots$ because from the first we have that $\omega_{0}<1$. Thus we proved that the cases $n=N_{0}(M) \geq v, M=R(N)$ will appear in set $D$ statistically with frequencies $\omega_{v}>(1 / 2)^{v}, v \in N$ and with a security levels $\varepsilon=1$ (i.e. 100\%). Combining the relations $\omega_{0}+\omega_{2}+\cdots=1$ and (113) we have that

$\omega_{0}<\omega_{1}<\omega^{*}=\omega_{2}+\omega_{3}+\cdots$, and then $\omega^{*}=\omega_{2}+\omega_{3}+\cdots>1 / 3>1 / 4$, with $\varepsilon=1$. Therefore we result that in any case more than the $1 / 4$ of the elements $\delta_{M}$ of $D$ satisfies the necessary condition $n=N_{M 0} \geq 2$ of the relation (109) because of (13). And thus finally we conclude that: "The relation (109) is valid to a part $\omega^{*}>1 / 4$ of the set $D^{\prime \prime}$. This is a useful Proposition: (121).

Defining as $\operatorname{Pr}_{v}=\{n \geq v>0\}$, the $\operatorname{Pr}_{v}\left[(1 / 2)^{v} \backslash \backslash D\right)$ will be valid.

3) The number of the primes in $\delta_{M}$ tends to infinity as $M$ tends to infinity that is $\lim _{M \rightarrow \infty}(n)=\infty$. Indeed, according to the previous (2) we conclude that $\forall \mu>0$ there is a population $\operatorname{Pop}_{\mu}$ from $\delta_{M}$ with non-zero occurrence frequency $\omega_{\mu}>(1 / 2)^{\mu}$, where each of these $\delta_{M}$ of $P_{0} p_{\mu}$ will has a number (multitude) of prime numbers $n=N_{0}(M) \geq \mu$. So we proved that the number of primes $n$ in RSIs tends to the infinite. Proposition: (122).

Based on the relation (109) and the proposition (121), to be valid the hypothe- 
sis that "the twin's multitude in $N$ is infinite", that is, the twins are infinite in the infinite number of RSIs, it is enough to be valid:

$$
\sum_{M=1}^{M=\infty}\left[P_{M}\right]^{2}=\infty,
$$

because from the counting of twins (5) and using the (109) with the proposition (121) we will have:

$$
R=\sum_{M=1}^{M=\infty} P_{M 0, \text { twin }}=\left[\omega^{*}\right] \frac{1}{2} \sum_{M=1}^{M=\infty}\left[P_{M}\right]^{2}>\frac{1}{4} \frac{1}{2} \sum_{M=1}^{M=\infty}\left[P_{M}\right]^{2}=\frac{1}{8} \sum_{M=1}^{M=\infty}\left[P_{M}\right]^{2}
$$

As we promised, we will repeat the calculations from the relation (4.16) of the first published article A until its relation (4.22), making the change dictated by the relation (104) etc. We will keep the same symbols of the article A in counting of below relations, reminding that $P_{v}=p(v)$ in the article A based on the following relation (4.17) is now the $P_{M 0}$ of RSI in the above, and the $\bar{P}_{v}=P_{M}(1)$ corresponds to the default VSI 1 of (81) and finally the $P_{M}$ is the probability of a prime in HVSI 1, 2. Also below we don't use the (4.19) of the article A because this relation refers to twin probability that we replaced by the correct (3) as we said. In article A we had the (4.16):

$$
P_{M 0}=p(v)>P_{M}=\prod_{j=1}^{M_{v}} F_{j}=\left(1-\rho_{1}\right)\left(1-\rho_{2}\right) \cdots\left(1-\rho_{M}\right)
$$

And now due to the (105) and (104) for HVSI 1, 2 it is true the (4.17) of the article A:

$$
P_{M 0}>P_{M}=\frac{1}{2} \prod_{j=2}^{M}\left(1-\frac{2}{q_{j}}\right), \forall M \in N
$$

In this way an interesting scenario occurs, a sequence of inequalities:

$$
\begin{aligned}
P_{M 0} & >P_{M}=\frac{1}{2} \prod_{j=2}^{M_{v}} F_{j}=\left(1-\frac{1}{2}\right)\left(1-\frac{2}{3}\right)\left(1-\frac{2}{5}\right)\left(1-\frac{2}{7}\right) \cdots\left(1-\frac{2}{q_{M}}\right) \\
& =\frac{1}{2}\left(\frac{1}{3} \frac{3}{5} \frac{5}{7} \frac{9}{11} \frac{11}{13} \frac{15}{17} \cdots \frac{q_{M-1}-2}{q_{M-1}} \frac{q_{M}-2}{q_{M}}\right) \\
& >\frac{1}{2}\left(\frac{1}{3} \frac{3}{5} \frac{5}{7}\left(\frac{7}{8} \frac{8}{9}\right) \frac{9}{11} \frac{11}{13}\left(\frac{13}{14} \frac{14}{15}\right) \frac{15}{17} \ldots \frac{q_{M-1}-2}{q_{M-1}}\left(\frac{q_{M-1}}{q_{M-1}+1} \cdots \frac{q_{M}-3}{q_{M}-2}\right) \frac{q_{M}-2}{q_{M}}\right) \\
& =1 /\left(2 q_{M}\right), \forall v \in \delta_{M}, \forall M \in N
\end{aligned}
$$

Particularly, all the successive fractions of the type $v /(v+1)$ were inserted in brackets (...) exactly where they were missing, which creates a more enhanced inequality. The last arose after the erasing of the equal numerators and denominators. Consequently for the true function $p(v)$, that defines the exact number of prime numbers in the random RSI $\delta_{M}$, the result will be the (4.18) of article A:

$$
\begin{aligned}
& \bar{P}_{v}=P_{M}(1) \geq P_{M 0}=p(v)=P(v)>P_{M} \\
& >1 /\left(2 q_{M}\right) \geq 1 /(2 \sqrt{v}), \quad \forall v \in \delta_{M}, \forall M \in N
\end{aligned}
$$

The last inequality from the above five inequalities of the relation (126) de- 
rives obviously from the relation $q_{M}^{2} \leq v=\alpha_{v}<q_{M+1}^{2}$ that refers to the natural numbers $v$ of the silver interval, whilst the others inequality of (126) derives from everything that was mentioned before for the consequences from the proposition (71) concerning the limits of $b_{s}(M)$.

Thereupon, for the natural number $a_{v}=v$ of the RSI $\delta_{M}$, the relation (126) with the above relations (109), (121), (123) results to (4.20) of A:

$$
R_{M}=\sum_{v=q_{M}^{2}}^{q_{M+1}^{2}-1} P_{M 0, \text { twin }}=\left[\omega^{*}\right] \frac{1}{2} \sum_{v=q_{M}^{2}}^{q_{M+1}^{2}-1}\left[P_{M}\right]^{2}>\frac{1}{32} \sum_{v=q_{M}^{2}}^{q_{M+1}^{2}-1} \frac{1}{v}
$$

Hence, the infinity multitude of the silver intervals one has the (4.21) of A:

$$
R=\sum_{M=1}^{\infty} R_{M}>\frac{1}{32} \sum_{M=1}^{\infty}\left(\sum_{v=q_{M}^{2}}^{q_{M+1}^{2}-1} \frac{1}{v}\right)=\frac{1}{32} \sum_{v=4}^{v=\infty} \frac{1}{v}=\left(\frac{1}{4}+\frac{1}{5}+\frac{1}{6}+\frac{1}{7}+\frac{1}{8}+\cdots\right)
$$

However, the second part of (128) becomes infinity because the brackets are the result of the subtraction of a finite multitude of terms from the known harmonic series, that as we know, it becomes infinity [(4.22) of article A)]:

$$
\frac{1}{1}+\frac{1}{2}+\frac{1}{3}+\frac{1}{4}+\frac{1}{5}+\frac{1}{6}+\cdots+\frac{1}{v}+\cdots=\infty
$$

Therefore, it was proven that the wanted multitude of the twin prime numbers in the infinite multitude of natural numbers will also be infinite.

"The dark paths (intervals) of infinity" in Section 5 of the A article is also a second method of solving this twin's problem applying the (126), (125) which will based on (104), (105) which we proved here in a clear way.

\section{Conclusions}

1) The main conclusion of the analysis is that the hypothesis that "twins are infinite" in $\mathrm{N}$ is correct. The evidentiary method examined all cases of overlapping bands and relied on the comparison between a random RSI and the corresponding HVSI 1, 2 thus exploiting on the basis of mathematical causality the catholic (or general or universal) information of the statistical distribution of twins until to infinity, based on the propositions (31), (32), thus excluding the case of accidental deviation of the calculations based on strict definitions deriving from mathematical necessity "that in mathematics nothing happens without a cause". Because of the unique definition of statistical counting of twins, that is the case.

2) The length of the RSI that is infinitely distant from the first RSI becomes statistically infinite as well as the number of its prime numbers becomes statistically infinite.

3) A numerical control method (insignificant for our proof) in the first 299 shows the arithmetical results in Table 2, where we see the very small number of twins of HVSIs 1, 2 in comparison to the corresponding RSIs.

Figure 3 shows the graph $d-n$, where the natural number $\mathrm{n}$ of horizontal axis represents the tens from silver intervals. We observe that to the initials 299 HVSI 1, 2 the fractions $\mathrm{d}=\mathrm{RT}(\mathrm{i}) / \mathrm{HT}(\mathrm{i})$ which are made from the average multitude 


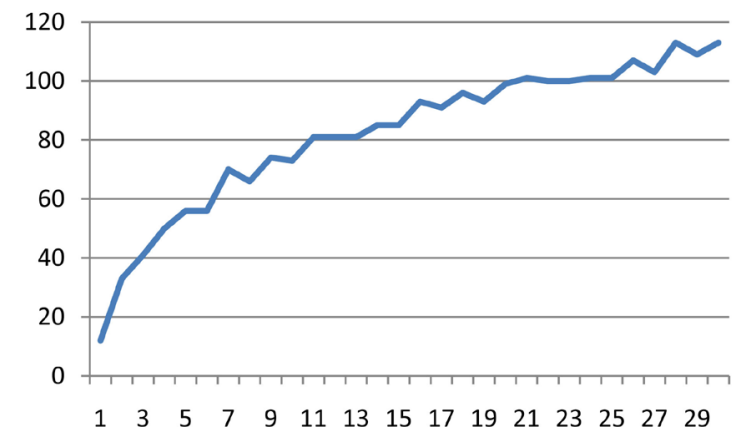

Figure 3. Graph $(d-n)$ of silver intervals.

Table 2. Correlation between different types of silver intervals.

\begin{tabular}{ccccc}
\hline & $\begin{array}{c}\text { Multitude of } \\
\text { Primes }\end{array}$ & $\begin{array}{c}\text { Multitude } \\
\text { of Twins }\end{array}$ & $\begin{array}{c}\text { Fraction } \\
\text { Primes/Twins }\end{array}$ & $\begin{array}{c}\text { Silver Interval } \\
\text { Multitude }\end{array}$ \\
\hline RSI & 279,791 & 26,592 & 10.5 & 299 \\
HVSI 1, 2 & 33,166 & 289 & 114 & 299 \\
VSI $g=1$ & 314,728 & 25,144 & 12.5 & 299 \\
\hline
\end{tabular}

$\mathrm{RT}(\mathrm{i})$ of twins per 10 respective RSI divided by the average multitude HT(i) of twins per $\mathrm{n}=10$ HVSI 1,2 , is increasing starting from about 10 until a value about 110. The increment of one order of magnitude between 10.5 (or 12.5) and 114 into Table 2 due to the relation $P_{\text {twin }}=P_{\text {prime }}^{2}$ that is $279,791 / 33,166$ is about 10.

4) No one of 299 cases gave twin's multitude of HVSI 1, 2 greater than the twin's multitude of the corresponding RSI. Also the $\mathrm{d}$ had the above great values with an increment as the $M$ was increasing which strengthens the inequalities of the proof. The arithmetical results are only an accompanied picture and nothing more in the previous proof. But the arithmetical finite experiments some times are useful because they indicate hidden cases and give birth new ideas indicating the way of research like every human experiment and experience.

5) Also the inequality (4.5) of article A has been controlled successfully in all these first 299 silver intervals.

\section{Conflicts of Interest}

The author declares no conflicts of interest regarding the publication of this paper.

\section{References}

[1] Papadopoulos, P. (2019) A Solution to the Famous “Twin's Problem”.

[2] Papadopoulos, P. (2015) The Twin of Infinity and the Riemann Conjecture. Ziti Edition, 661.

[3] Wiener, N. (1948) Cybernetics: Or, Control and Communication in the Animal and the Machine. MIT University Press, Cambridge, MA, 212.

[4] Spiegel, M.R. (1975) Probability and Statistics. McGraw, Hill, New York, 372. 


\section{Appendix}

\section{Nomenclatures}

1) a) Real Silver Interval (RSI): $\delta_{M}=\left[q_{M}^{2}, q_{M+1}^{2}\right)$ with arithmetical length

$$
d_{M}=q_{M+1}^{2}-q_{M}^{2}, \quad M \in N,
$$

where $q_{M}, q_{M+1}$ are two successive prime numbers. This is also defined in Sections $1 \mathrm{~A}$ of Introduction, also in 2, and it is referred in Section 4, e.g. relation (80). Sub sequent of all types of Silver Intervals (SI) is: $Y_{M}=\left\{q_{1}, q_{2}, q_{3}, \cdots, q_{M}\right\}$. Defined in the Section 1B and also referred at the beginning of the Section 2. b) And for the Sections 3, 5 etc., we define as random choice from a set $A$ and under a Catholic Information's restriction $C I R$ the random selection of an element under this general restriction $C I R: a_{i}=R(A \| C I R)$. In case with infinite number of elements we expand the finite definition to the infinite letting the number $N$ of the elements of $A$ tends to the infinite. Also we define as Gold Interval (GI): $\Delta_{N}=\left[1, q_{1} q_{2} \cdots q_{N}\right]$, with its sub sequent $X_{N}=\left\{q_{1}, q_{2}, q_{3}, \cdots, q_{N}\right\}$.

2) Probability to happening the event $e_{1}: p\left(e_{1}\right)$. And also the probability to happening the event $e_{1}$ under the condition to be happened another event $e_{2}$ : $p\left(e_{1} \| e_{2}\right)$. And their independence will be $p\left(e_{1} \| e_{2}\right)=p\left(e_{1}\right)$.

3) Virtual Silver Interval: [VSI $g$ ] or $\delta_{M}^{\text {vir }}(g)$. This is defined in Sections $1 \mathrm{~B}, 2$ and it also is referred in Section 4, e.g. the relations (24), (82). The special VSI $g=1$ or $\delta_{M}^{\text {vir }}(1)$ we call as default VSI.

4) Hybrid Virtual Silver Interval 1, 2: HVSI 1, 2. This is defined in Section 4, e.g. the relation (104).

5) $n=N_{M 0}, n_{t}=N_{M 0, t w i n}$ are the multiyudes of the primes ant the twins in a Random RSI [or symbolically $R\left(\delta_{M}\right)$ ] correspondingly. And $N_{M}, N_{M \text {,twin }}$ are the multiudes of the primes and the twins in the corresponding HVSI 1, 2. Also $P_{M 0}, P_{M 0, t w i n}$ are the probabilites to find a prim and a twin in a random palce $v$ of a $R\left(\delta_{M}\right)$, see relation (80). And $P_{M}, P_{M, t w i n}$ are the probabilites to find a prim and a twin in a random palce of the corresponding HVSI 1, $2 R\left(\delta_{M}\right)$, see relation (104).

6) The Band $b_{s}(M)$ is the subinterval of all the multiples [moving eraser: ( $q_{s}$-multiple)] of some prime number $q_{s} \in Y_{M}$, defined between the two limits $\theta_{s}(1, M), \theta_{s}(\tau, M)$ of their space $\left[b_{s}(M)\right]$ in all types of Silver Intervals:

$$
b_{s}(M)=\left[\theta_{s}(1, M), \theta_{s}(\tau, M)\right] \text {. }
$$

This is defined in Sections 1B, 2.

7) "Real clear density of deletion" and "Virtual clear density of deletion". These are defined in Section $1 \mathrm{~B}$ and in the Section 2, e.g. the $\rho=U_{M}(g)$ of the relation (28).

8) "Catholic Information". This is referred below in "The steps", and it is defined at the beginning of the Section 3.

9) We define two events of divisibility $v \mid \mu$ and not divisibility $v$, not $\mid \mu$, where a natural number $v$ divides and not devides another natural number $\mu$ 
correspondingly. These definitions exist also in Section 3.

10) The inequality (3.2) or (4.5) of article A for RSI $\delta_{M}$ is important:

$$
d_{M}=q_{M+1}^{2}-q_{M}^{2}>4 q_{M} \geq 4 q_{s}, \forall s=1,2,3,4, \cdots, M \text { and } \forall M>1
$$

This is used e.g. in Section 4 for proving the (74), (75), (77) etc. 